\title{
In vitro behavior of tendon stem/progenitor cells on bioactive electrospun nanofiber membranes for tendon-bone tissue engineering applications
}

This article was published in the following Dove Press journal: International Journal of Nanomedicine

\author{
Yucheng $\operatorname{Lin}^{1-3, *}$ \\ Lu Zhang ${ }^{4, *}$ \\ Nancy Q Liu ${ }^{5}$ \\ Qingqiang Yao ${ }^{1,2}$ \\ Ben Van Handel ${ }^{5}$ \\ Yan $\mathrm{Xu}^{1,2}$ \\ Chen Wang ${ }^{3}$ \\ Denis Evseenko ${ }^{5}$ \\ Liming Wang ${ }^{1,2}$
}

'Department of Orthopaedic Surgery, Nanjing First Hospital, Nanjing Medical University, Nanjing, Jiangsu, People's Republic of China; ${ }^{2}$ Digital Medicine Institute, Nanjing Medical University, Nanjing, Jiangsu, People's Republic of China; ${ }^{3}$ Department of Orthopaedic Surgery, Zhongda Hospital, School of Medicine, Southeast University, Nanjing, Jiangsu, People's Republic of China;

${ }^{4}$ Department of Anesthesiology,

Women's Hospital of Nanjing Medical

University, Nanjing Maternity and Child

Health Care Hospital, Nanjing, Jiangsu,

People's Republic of China; ${ }^{5}$ Department

of Orthopaedic Surgery, University of

Southern California (USC), Los Angeles, CA, USA

*These authors contributed equally to this work

\begin{abstract}
Purpose: In order to accelerate the tendon-bone healing processes and achieve the efficient osteointegration between the tendon graft and bone tunnel, we aim to design bioactive electrospun nanofiber membranes combined with tendon stem/progenitor cells (TSPCs) to promote osteogenic regeneration of the tendon and bone interface.
\end{abstract}

Methods: In this study, nanofiber membranes of polycaprolactone (PCL), PCL/collagen I (COL-1) hybrid nanofiber membranes, poly(dopamine) (PDA)-coated PCL nanofiber membranes and PDA-coated PCL/COL-1 hybrid nanofiber membranes were successfully fabricated by electrospinning. The biochemical characteristics and nanofibrous morphology of the membranes, as well as the characterization of rat TSPCs, were defined in vitro. After coculture with different types of electrospun nanofiber membranes in vitro, cell proliferation, viability, adhesion and osteogenic differentiation of TSPCs were evaluated at different time points.

Results: Among all the membranes, the performance of the PCL/COL-1 (volume ratio: $2: 1 \mathrm{v} / \mathrm{v}$ ) group was superior in terms of its ability to support the adhesion, proliferation, and osteogenic differentiation of TSPCs. No benefit was found in this study to include PDA coating on cell adhesion, proliferation and osteogenic differentiation of TSPCs.

Conclusion: The PCL/COL-1 hybrid electrospun nanofiber membranes are biocompatible, biomimetic, easily fabricated, and are capable of supporting cell adhesion, proliferation, and osteogenic differentiation of TSPCs. These bioactive electrospun nanofiber membranes may act as a suitable functional biomimetic scaffold in tendon-bone tissue engineering applications to enhance tendon-bone healing abilities.

Keywords: electrospinning, TSPCs, nanomaterial, biomimetic scaffold, osteogenic differentiation, tendon-bone healing

\section{Introduction}

Tendon or ligament injuries are common in both the workplace and sports. Over 30 million tendon-related procedures take place worldwide per year, accompanied by a large economic burden consisting of both medical expenses and lost productivity. ${ }^{1}$ Anterior cruciate ligament (ACL) rupture is one of the most frequent and debilitating tendon or ligament injuries in the knee joint. Moreover, ACL injury causes instability of the knee joint, leading to the subsequent development of degenerative diseases. $^{2}$

Reconstructive surgery with tendon grafts is still one of the most commonly used ACL injury treatments. However, due to the poor healing capacity of the
Correspondence: Liming Wang Department of Orthopaedic Surgery, Nanjing First Hospital, Nanjing Medical University, 68 Changle Road, Nanjing, Jiangsu, People's Republic of China Tel +86 I 8951670968

Email limingwang99@yahoo.com 
tendon and the lack of a suitable biointerface between the tendon graft and bone tunnel for efficient integration, healing after ACL reconstruction is slow and insufficient. ${ }^{3}$ Tendon autograft requires 3 years for sufficient integration with host bone after ACL reconstruction in humans, and the graft can be pulled out even 12 weeks after ACL reconstruction in animals. ${ }^{4,5}$ Hence, during the post-operative period of ACL reconstruction the "weak point" is the interface between the tendon graft and the tunnel bone, which largely determines the security and success of tendon-bone healing. ${ }^{6}$

It is vital to explore better strategies to induce functional integration and augment tendon-bone healing abilities. Numerous studies have proposed approaches to interface regeneration between the tendon and bone, such as the use of osteoinductive growth factors, enveloping the grafts with periosteum or polymeric biomaterials, stem cell-based therapies, and tissue-engineered scaffolds. ${ }^{7-10}$ Although no standardized method has proven overly efficacious, constructs of stem cells with biodegradable scaffolds hold great promise for improving tendon-to-bone healing. ${ }^{11,12}$

Recently, nanofibrous scaffolds fabricated by electrospinning techniques have become increasingly popular in the field of tendon-bone tissue engineering. ${ }^{13}$ Electrospun nanofiber membranes are characterized by their biocompatible and biodegradable polymers, high porosity, high surface/volume ratio, and potential to mimic the hierarchical structure of extracellular matrix (ECM). These properties make electrospun nanofibers well-suited as scaffolds for tissue engineering applications. ${ }^{14}$ A rich variety of materials (both natural and synthetic) have been used in electrospinning processes, such as polycaprolactone (PCL), poly-1-lactide (PLLA), poly(D, 1-lactide-co-glycolide) (PLGA), collagen and gelatin. ${ }^{15-19}$

PCL is a biodegradable and biocompatible polymer commonly used for tissue engineering scaffold, which has been approved by the US Food and Drug Administration for various medical applications. Nevertheless, the hydrophobicity of pure PCL makes the scaffold adverse to cell adhesion, growth and differentiation. ${ }^{20}$ The functionalization of nanofibers in tissue engineering scaffolds can be achieved mainly through two strategies: one approach is by blending natural polymers or bioactive molecules including collagen-1(COL-1) and nanohydroxyapatite to fabricate the hybrid scaffolds, while another is through surface coating with bioactive agents after electrospinning to obtain surface-modified scaffolds. ${ }^{21}$ In this study, we chose COL-1, a natural polymer with excellent biocompatibility, ${ }^{18}$ to overcome the shortcomings of using natural or synthetic polymers alone in tendon-bone tissue engineering. Concurrently, we used poly(dopamine) (PDA), an organic compound inspired by the composition of adhesive proteins in mussels, ${ }^{22}$ which could promote cell adhesion and regulate stem cells differentiation on various substrates to modify the surface of electrospun nanofiber membranes. ${ }^{23,24}$ The strategy of using PDA coating was welcomed in tissue engineering for its mild coating procedure, beneficial interaction with cells and convenient advantages. It was reported that PDA deposition not only promoted the adhesion of osteoblasts but also the proliferation of osteoblasts in biodegradable polymers. ${ }^{25}$ And enhanced bone regeneration was occurred in a PDA-coated surface of PLLA nanofibers compared with the fibers without coating. ${ }^{26}$ Therefore, in the present study, we used PCL blending with COL-1 to fabricate hybrid scaffolds of synthetic and natural polymers and PDA-coated surfacemodified scaffolds simultaneously. We aim to compare the superiority of these two electrospun membrane functionalization strategies in order to find out which method is more conducive to cell adhesion, proliferation, and differentiation.

In the past few years, a unique type of mesenchymal stem cells (MSCs) has been identified from tendons of different species including human, mouse, rat, rabbit and horse, called tendon stem/progenitor cells (TSPCs). ${ }^{27-30}$ Using TSPCs as the seed cells for tendon-bone tissue engineering might be advantageous considering that the tendon milieu is an ideal and familiar environment which might promote the differentiation of the transplanted cells. ${ }^{31}$ Moreover, TSPCs exhibited higher clonogenicity, cell proliferation, and tenogenic/chondrogenic/osteogenic differentiation potential compared to bone marrow mesenchymal stem cells (BMSCs), suggesting that they could be a better cell source for tendon-bone healing. ${ }^{27,32}$

The aim of this work was to fabricate bioactive electrospun nanofiber membranes combined with TSPCs to promote tendon-bone interface integration. We hypothesized that the nanofibrous membranes could act as a functional biomimetic scaffold to enhance cell adhesion, proliferation and osteogenic differentiation of TSPCs in vitro. Our findings provide support for further in vivo studies in tendon-bone tissue engineering applications.

\section{Materials and methods}

\section{Fabrication of nanofiber membranes}

Nanofiber membranes were prepared by a routine electrospinning method. ${ }^{33}$ For PCL membranes: PCL (molecular 
weight $80 \mathrm{kDa}$, Sigma-Aldrich, USA) was dissolved in 1,1,1,3,3,3-hexafluoro-2-propanol (HFP, Aladdin, China) at a concentration of $10 \%(\mathrm{w} / \mathrm{v})$ and was electrospun using a high voltage power supply at $12 \mathrm{kV}$ potential between the solution and the grounded surface. The solution was delivered with a $20 \mathrm{~mL}$ polypropylene syringe through a 16 gauge blunt tip needle at a flow rate of $1.0 \mathrm{~mL} / \mathrm{h}$ using a syringe pump. Fibers were collected onto a grounded aluminum foil plate at a distance of $20 \mathrm{~cm}$ from the syringe tip. For PCL/ COL-1 hybrid membranes: PCL and COL-1 (derived from pig skin, molecular weight 80-100 kDa, Kele, China) were dissolved in HFP at concentrations of $10 \%$ and $7 \%(\mathrm{w} / \mathrm{v})$ respectively. And then a series of PCL/COL-1 blend solutions $(4 / 1 \mathrm{v} / \mathrm{v}, 2 / 1 \mathrm{v} / \mathrm{v}, 1 / 1 \mathrm{v} / \mathrm{v}, 1 / 2 \mathrm{v} / \mathrm{v})$ were prepared by mixing each solution and delivering them by the same procedure as above. The distance between the needle tip and ground electrode remained $20 \mathrm{~cm}$, and a positive voltage of 20 $25 \mathrm{kV}$ was applied to the polymer solutions. For PDA surface coating membranes, the coating method was used as previously described. ${ }^{22}$ In brief, the electrospun nanofiber membranes were immersed in PDA (H8502, Sigma-Aldrich, USA) which was dissolved in a $\mathrm{pH} 8.5,10 \mathrm{mM}$ Tris buffer $(2 \mathrm{mg} / \mathrm{mL}$ ) solution for $1 \mathrm{~h}$ with shaking and unbound PDA was removed by washing several times with distilled water. All procedures were performed at room temperature.

\section{Characterization of nanofiber membranes Scanning electron microscopic observation of nanofibers}

Electrospun membranes were sputter-coated with gold (JEOL JFC-1600 Auto Fine Coater, Japan) and nanofiber morphologies were examined by scanning electron microscopy (SEM, Model S-4800, Hitachi Co. Ltd., Japan) at an accelerating voltage of $5 \mathrm{kV}$. The diameter of the fiber was measured with Image $\mathbf{J}$ software (National Institutes of Health, USA). The average and standard deviation of fiber diameters were calculated from over 50 random measurements per image.

\section{Porosity measurements and dissolvability test}

Mercury intrusion porosimetry was used to evaluate the porosity of the electrospun nanofiber membranes as described previously. ${ }^{34}$ Measurements of the porosity of samples in each group were performed and analyzed with Micromeritics (Autopore IV-9500, USA).

Electrospun membranes were cut into $6 \mathrm{~cm} \times 6 \mathrm{~cm}$ pieces, and then accurately weighed and placed in phosphate-buffered saline (PBS) $\left(\mathrm{pH}=7.4 ; 37^{\circ} \mathrm{C}\right)$ for $1 \mathrm{~h}$.
Membranes were then removed from PBS and dried in the drying oven $\left(50^{\circ} \mathrm{C}\right)$ for $5 \mathrm{~h}$. The dehydrated samples were weighed again and the water-dissolved rate was determined as the percentage of weight loss $\left(\mathrm{W}_{\mathrm{L}}\right)$ according to the equation.

$$
\mathrm{W}_{\mathrm{L}}(\%)=\left[\left(\mathrm{W}_{0}-\mathrm{W}\right) / \mathrm{W}_{0}\right] \times 100
$$

Where $\mathrm{W}_{0}$ is the initial weight of membrane in its dry state and $\mathrm{W}$ is the weight of membrane after dissolution. Three samples were tested in each group.

\section{Water contact angles of nanofiber membranes}

The water contact angles of different types of membranes were measured by a water contact angle analysis system (OCA15EC, Dataphysics, Germany). Samples of dimensions $1.5 \times 1 \mathrm{~cm}$ were cut out from the membranes and placed on the plate. One drop of distilled water was added to their surfaces. Contact angles from $0^{\circ}$ to $30^{\circ}$ were regarded as highly hydrophilic, $30^{\circ}$ to $90^{\circ}$ as hydrophilic, and $90^{\circ}$ to $150^{\circ}$ as hydrophobic. The results are calculated from three independent samples.

\section{Fourier-transform infrared analysis}

The chemical components of PCL membranes, PCL/COL1 membranes and PDA surface coating membranes were analyzed by attenuated total reflectance-Fourier transform infrared spectroscopy (ATR-FTIR) (spectrum 100, Perkin Elmer Inc., USA) in the range of $400-4000 \mathrm{~cm}^{-1}$.

\section{Isolation and culture of rat TSPCs}

Three 8-week-old Sprague-Dawley rats, weighing 250$280 \mathrm{~g}$, were used. Animal experiments were approved by the Animal Research Ethics Committee of Nanjing Medical University and followed the guidelines of the Chinese Society of Laboratory Animals on animal welfare. The procedures for the isolation of TSPCs from rat patellar tendon have been well established. ${ }^{35}$ Briefly, the mid-substance of patellar tendons were excised from both limbs of each rat. Peritendinous connective tissue was carefully removed and the samples were stored in sterile PBS. The tissues were minced into $1.5 \times 1.5 \times 1.5 \mathrm{~mm}^{3}$ pieces, digested with type I collagenase $(5 \mathrm{mg} / \mathrm{mL}$; SigmaAldrich, USA) for $2.5 \mathrm{~h}$ at $37^{\circ} \mathrm{C}$, and then passed through a $70 \mu \mathrm{m}$ cell strainer (Becton Dickinson, USA) to yield a single-cell suspension. The released cells were washed in PBS by centrifugation at $300 \mathrm{~g}$ for $5 \mathrm{~min}$ and resuspended in a complete culture medium [Dulbecco's Modified Eagle Medium (DMEM), 10\% fetal bovine serum, $100 \mathrm{U} / \mathrm{mL}$ penicillin, $100 \mathrm{mg} / \mathrm{mL}$ streptomycin] (all from Invitrogen 
Corporation, USA). The isolated nucleated cells were plated at low cell density ( 50 nucleated cells $/ \mathrm{cm}^{2}$ ) for the isolation of stem cells and cultured at $37^{\circ} \mathrm{C}, 5 \% \mathrm{CO}_{2}$ to form colonies. At day 2, the cells were washed with PBS to remove the nonadherent cells. At day 7, they were trypsinized and mixed together as passage 0 (P0). Culture medium was changed every 3 days. Cells from passages 3-4 (P3-P4) were used for the remaining experiments. After the identification of TSPCs from three rats, TSPCs were pooled together for seeding the cells to electrospun nanofiber membranes in vitro.

\section{Identification of TSPCs Colony formation assay}

The nucleated cells derived from the patellar tendons of three rats were plated at 50 cells $/ \mathrm{cm}^{2}$ in $20 \mathrm{~cm}^{2}$ dishes and cultured for 10 days. Cells were washed with PBS twice, fixed with $4 \%$ paraformaldehyde for $10 \mathrm{~min}$ and then stained with $0.5 \%$ crystal violet (Sigma, USA) for $20 \mathrm{~min}$ to show the number and morphology of cell colonies.

\section{Flow cytometry assay}

After TSPCs reached $80 \%$ confluence at P3, cells were rinsed with PBS and treated with $0.25 \%$ trypsin-EDTA for collection and the resulting cell suspension adjusted to a concentration of $1 \times 10^{6}$ cells $/ \mathrm{mL}$ in ice-cold PBS (with $10 \%$ fetal bovine serum and $1 \%$ sodium azide). The cells were incubated with fluorochrome-conjugated primary antibodies against CD31 (ab33858, Abcam, USA), CD34 (sc-7324; Santa Cruz Biotechnology, USA), CD44 (ab23396, Abcam, USA), CD90 (ab33694, Abcam, USA) or corresponding isotype control (BD Biosciences, USA) in dark at $4{ }^{\circ} \mathrm{C}$ for $30 \mathrm{~min}$. After washing with PBS at $400 \mathrm{~g}$ for $5 \mathrm{~min}$, the stained cells were re-suspended in $500 \mu \mathrm{L}$ of ice-cold PBS (with $10 \%$ fetal bovine serum and $1 \%$ sodium azide) and subjected to fluorescence activated cell sorter (FACS) analysis (FACS Calibur, Becton Dickinson, USA). Triplicates of cells from three rats were examined in this experiment.

\section{In vitro multi-lineage differentiation potential}

Osteogenic differentiation: TSPCs were plated at $4 \times 10^{3}$ cells $/ \mathrm{cm}^{2}$ in a 6 -well plate and cultured in the complete culture medium until the cells reached $80 \%$ confluence. They were then incubated in complete medium or osteogenic induction medium, which was supplemented with $1 \mathrm{nM}$ dexamethasone, $50 \mathrm{mM}$ ascorbic acid, and $20 \mathrm{mM}$ $\beta$-glycerolphosphate (all from Sigma-Aldrich, USA) at
$37{ }^{\circ} \mathrm{C}, 5 \% \mathrm{CO}_{2}$ for 21 days. The mineralization of TSPCs was assessed by Alizarin red S staining. Briefly, the cells were washed with the PBS, fixed in $4 \%$ paraformaldehyde for $20 \mathrm{~min}$ and stained with $0.5 \%$ alizarin red S (pH 4.1, Sigma-Aldrich, USA) for $10 \mathrm{~min}$.

Adipogenic differentiation: TSPCs were plated at $4 \times 10^{3}$ cells $/ \mathrm{cm}^{2}$ in a 6 -well plate and cultured in the complete culture medium until the cells reached complete confluence. The medium was replaced with complete medium or adipogenic medium, which was supplemented with $500 \mathrm{nM}$ dexamethasone, $0.5 \mathrm{mM}$ isobutylmethylxanthine, $50 \mu \mathrm{M}$ indomethacin, and $10 \mu \mathrm{g} / \mathrm{mL}$ insulin (all from SigmaAldrich, USA). The cells were cultured for another 21 days for the detection of oil droplets by oil red-O staining. Briefly, the cells were washed with PBS twice then fixed in $4 \%$ paraformaldehyde for $20 \mathrm{~min}$ and finally incubated with $0.3 \%$ fresh oil red-O solution (Sigma-Aldrich, USA) for $1 \mathrm{~h}$.

Chondrogenic differentiation: For chondrogenic differentiation, a 3D-pellet culture system was used as described previously. ${ }^{29} 4 \times 10^{5}$ cells were transferred into a $15-\mathrm{mL}$ conical polypropylene tube and centrifuged at $450 \mathrm{~g}$ for $10 \mathrm{~min}$ to form micro mass pellets. Cells were then cultured in complete medium or chondrogenic induction medium, consisting of complete medium supplemented with $10 \mathrm{ng} / \mathrm{mL}$ transforming growth factor- $\beta 3$ (R\&D Systems, USA), $500 \mathrm{ng} / \mathrm{mL}$ bone morphogenetic protein-2 (R\&D Systems,USA), $10^{-7} \mathrm{M}$ dexamethasone, $50 \mathrm{mg} / \mathrm{mL}$ ascorbate-2-phosphate, $40 \mathrm{mg} / \mathrm{mL}$ proline, $100 \mathrm{mg} / \mathrm{mL}$ pyruvate (all from Sigma-Aldrich, USA), and 1:100 diluted ITS+Premix $(6.25 \mathrm{mg} / \mathrm{mL}$ insulin, $6.25 \mathrm{mg} / \mathrm{mL}$ transferrin, $6.25 \mathrm{mg} / \mathrm{mL}$ selenous acid, $1.25 \mathrm{mg} /$ $\mathrm{mL}$ bovine serum albumin, and $5.35 \mathrm{mg} / \mathrm{mL}$ linoleic acid) (Becton Dickinson, USA) at $37{ }^{\circ} \mathrm{C}, 5 \% \mathrm{CO}_{2}$. The chondrogenic medium was changed every 3 days. At day 21 , the pellet was fixed and assessed by alcian blue staining as described below. The cell pellet was fixed in $4 \%$ paraformaldehyde, dehydrated, and embedded in paraffin. Sections were cut at a thickness of $5 \mu \mathrm{m}$ and were stained with $1 \%$ alcian blue solution (pH2.5, G1027, Servicebio technology, China) for 15 min after deparaffination and viewed using a Nikon Eclipse Ci microscope (Nikon DS-Fi2 imaging system, Japan).

\section{Co-culture of TSPCs and electrospun nanofiber membranes in vitro Cell proliferation}

The electrospun nanofiber membranes were trimmed to $5 \mathrm{~mm}$ in diameter by a punch and placed in a 96-well plate. Scaffolds were sterilized by immersing them in $70 \%$ 
ethanol for $30 \mathrm{~min}$, exposing them to UV light for $50 \mathrm{~min}$ on each side, and washing with sterile PBS. TSPCs were seeded in each well at $1 \times 10^{4} /$ well and cultured with complete culture medium. We have established a positive control group (cells cultured in normal 96 well plates) and a blank control group (medium only control wells without cells) in order to minimize experimental errors. After culture for 1, 3, 5 and 7 days, cell proliferation of TSPCs were measured with cell counting kit-8 assay (CCK-8, bimake, USA). Briefly, $20 \mu \mathrm{lCK}-8$ solution was added in the $200 \mu \mathrm{l}$ culture medium at each well and the cells were incubated at $37{ }^{\circ} \mathrm{C}, 5 \% \mathrm{CO}_{2}$ for $2 \mathrm{~h} .200 \mu \mathrm{L}$ of the resulting solution from each well was transferred to a new 96-well plate and the optical density value at $450 \mathrm{~nm}$ was recorded with a microplate reader (SpectraMax CMax Plus, MolecularDevices Inc., USA). There were five samples in each group at each time point.

\section{Cell viability}

The viability of TSPCs cultured in the membranes on day 1, 3, 5 was assessed using the Live/Dead Viability/ Cytotoxicity Kit (L3224, Molecular Probes, USA) according to the manufacturer's instructions. In brief, sterilized electrospun nanofiber membranes were placed in a 96-well plate and the cell density was $1 \times 10^{4}$ cells/well. Samples were washed with PBS and incubated in the dilute dye solution for $30 \mathrm{~min}$ at room temperature. After washing with PBS, these samples were observed and photographed using a laser scanning confocal microscope (LSCM, Zeiss LSM710, Carl Zeiss, Germany). Live cells were stained green, and the nucleus of dead cells appeared red $(n=3$ in each group).

\section{Scanning electron microscopic observation}

TSPCs were cultured as above in the membranes at $1 \times 10^{5}$ cells/well for 1,3 , and 5 days. For SEM observation, the samples were washed twice with PBS and fixed with $4 \%$ paraformaldehyde for $1 \mathrm{~h}$. They were then dehydrated in ethanol solution at concentrations of $50 \%, 70 \%, 80 \%$, $90 \%$ and $100 \%$ and finally dried in air. The samples were gold sputtered under vacuum and visualized under SEM (Model S-4800, Hitachi Co. Ltd., Japan) at $5 \mathrm{kV}(\mathrm{n}=3$ in each group).

\section{Fluorescence microscopy}

The cytoskeletal morphology of TSPCs grown on different types of membranes was analyzed by using F-actin staining. After $24 \mathrm{~h}$ cultivation, samples ( $\mathrm{n}=3$ in each group) were fixed with $4 \%$ paraformaldehyde for $15 \mathrm{~min}$, permeabilized with $0.1 \%$ Triton $\mathrm{X}-100$ for 5 min, blocked with 1\% BSA for $30 \mathrm{~min}$, stained with Rhodamine-phalloidin (AAT Bioquest, USA) for $30 \mathrm{~min}$ and counterstained with DAPI (KeyGEN, China) for $20 \mathrm{~min}$ at room temperature under dark conditions. The stained cells were observed using LSCM (Zeiss LSM710, Carl Zeiss, Germany). Images were exported into Image $\mathbf{J}$ and outlines were drawn around cells. Projected cell areas were determined for each cell.

\section{Quantitative real-time reverse transcription polymerase chain reaction ( $\mathrm{QRT}$-PCR)}

To evaluate the effect of nanofiber membranes on osteogenic differentiation, the mRNA expression of several genes was examined in TSPCs cultured on the scaffolds in normal media for 4, 7 and 14 days. TSPCs were harvested and homogenized for RNA extraction with RNeasy mini kit (74,104, Qiagen GmbH, Germany). The concentration of RNA was determined by measuring the optical density at 260/280 nm. RNA was reverse transcribed to cDNA using the First Strand cDNA kit (RR047A, Takara, Japan), following the manufacturer's protocol. PCR was performed using a Power SYBR Green PCR Master Mix (Invitrogen Corporation, USA) on a real-time PCR machine (ABI 7500 Plus, USA). Cycling conditions were denaturation at $95^{\circ} \mathrm{C}$ for $10 \mathrm{~min}, 40$ cycles at $95^{\circ} \mathrm{C}$ for $20 \mathrm{~s}$, optimal annealing temperature at $60^{\circ} \mathrm{C}$ for $45 \mathrm{~s}$ and finally at $60-95^{\circ} \mathrm{C}$ with a heating rate of $0.3^{\circ} \mathrm{C} / \mathrm{s}$. Expression of the target gene was normalized to that of the $\beta$-actin gene. Relative gene expression was calculated according to the $2^{-\Delta \Delta C T}$ formula. All primers were designed using primer 5.0 and are summarized in Table 1. Each real-time PCR was performed on at least 4 different experimental samples.

\section{Immunofluorescent staining}

To assess the osteogenic differentiation of TSPCs on membranes at the protein level, immunofluorescent staining assays for Collagen I alpha 2 (Col1a2), Osteocalcin (Ocn) and Runx-2 ( $\mathrm{n}=3$ in each group) were performed at day 7, 14 and 21 after co-culture in vitro. Briefly, the samples were fixed in $4 \%$ paraformaldehyde for $15 \mathrm{~min}$ and washed with PBS three times for $5 \mathrm{~min}$ each. The samples were permeabilized by incubation with $0.1 \%$ Triton-X100 in PBS for $10 \mathrm{~min}$ followed by incubation in 3\% BSA in PBS for $1 \mathrm{~h}$ to reduce nonspecific background staining. Next, these samples were treated with a primary antibody overnight at $4^{\circ} \mathrm{C}$, incubated with a secondary antibody for $1 \mathrm{~h}$ at room temperature and then 
Table I The primer sequences of the genes for qRT-PCR

\begin{tabular}{|l|l|l|l|}
\hline Gene & Primer & Sequence (5' to 3') & Gene bank identification \\
\hline$\beta$-actin & $\begin{array}{l}\text { Forward } \\
\text { Reverse }\end{array}$ & $\begin{array}{l}\text { ATCGTGGGCCGCCCTAGGCA } \\
\text { TGGCCTTAGGGTTCAGAGGGG }\end{array}$ & NM_03II44 \\
\hline Collal & $\begin{array}{l}\text { Forward } \\
\text { Reverse }\end{array}$ & $\begin{array}{l}\text { CATCGGTGGTACTAAC } \\
\text { CTGGATCATATTGCACA }\end{array}$ & NM_053356.I \\
\hline Ocn & $\begin{array}{l}\text { Forward } \\
\text { Reverse }\end{array}$ & $\begin{array}{l}\text { GGTGCAAAGCCCAGCGACTCT } \\
\text { GGAAGCCAATGTGGTCCGCTA }\end{array}$ & M23637 \\
\hline Runx-2 & $\begin{array}{l}\text { Forward } \\
\text { Reverse }\end{array}$ & $\begin{array}{l}\text { CCGATGGGACCGTGGTT } \\
\text { CAGCAGAGGCATTTCGTAGCT }\end{array}$ & XM_346016 \\
\hline Oct4 & $\begin{array}{l}\text { Forward } \\
\text { Reverse }\end{array}$ & $\begin{array}{l}\text { CATCTGCCGCTTCGAG } \\
\text { CTCAATGCTAGTCCGCTTTC }\end{array}$ & NM_001009I78.2 \\
\hline
\end{tabular}

Abbreviations: Collal, collagen I alpha I; Ocn, osteocalcin.

counterstained with DAPI (KeyGEN, China) for $20 \mathrm{~min}$ under dark conditions. Antibodies were used at the following concentrations: Anti-Col1a2 antibody (ab96723; Abcam, UK), 1:150; Anti-Ocn antibody (ab13420; Abcam, UK), $2 \mu \mathrm{g} / \mathrm{mL}$; Anti-Runx-2 antibody (ab192256; Abcam, UK), 1:1000; FITC-conjugated goat anti-rabbit IgG, 1:500 (Invitrogen, USA); Rhodamine-conjugated goat anti-mouse IgG, 1:500 (Invitrogen, USA). The stained samples were visually observed using LSCM (Zeiss LSM710, Carl Zeiss, Germany).

\section{Statistical analysis}

All the quantitative results were expressed as mean \pm standard deviation (SD). Statistical analysis was carried out by means of one-way analysis of variance (ANOVA). A $p$ value less than 0.05 was considered statistically significant.

\section{Results}

\section{Identification of characteristics of electrospun nanofiber membranes}

The PCL membranes and PCL/COL-1 membranes with four different PCL/COL-1 ratios were effectively fabricated by electrospinning. Figure $1 \mathrm{~A}-\mathrm{J}$ showed the SEM images of the nanofibers in different membranes. The average diameters of the nanofibers with different PCL/ COL-1 ratios $(4: 1,2: 1,1: 1,1: 2)$ were $613.12 \pm 203 \mathrm{~nm}$, $549.37 \pm 134 \mathrm{~nm}, 507.33 \pm 107 \mathrm{~nm}$, and $515.70 \pm 147 \mathrm{~nm}$, respectively. The average diameter of pure PCL nanofibers was $1207.94 \pm 167 \mathrm{~nm}$, which was much larger than the fibers of PCL/COL-1 membranes (Figure 1K). The porosity of nanofiber membranes ranged from $82.8 \%$ to $92.8 \%$, which was desirable for the scaffolds (Figure 1L). As shown in Figure 1M, PCL/COL-1(1:2) had a higher dissolvability $(19.04 \pm 2.9 \%)$ compared with PCL $(0.01$ $\pm 0.0 \%)$, PCL/COL-1(4:1) (1.80 $\pm 0.4 \%)$, PCL/COL-1(2:1) $(2.86 \pm 0.5 \%)$, and PCL/COL-1(1:1) $(3.18 \pm 0.8 \%)$.

In SEM images (Figure 2A-E), the surface of the fibers was smooth, while lumps of coated PDA were partially observed on the surface of nanofibers. As shown in Figure 2Q and R, ATRFTIR spectra for PCL-related and collagen-related stretching modes were observed for PCL nanofibers, PCL/COL-1 nanofibers and PDA coated nanofibers. Typical absorption bands for PCL of carbonyl stretching and C-O stretching were found at $1724 \mathrm{~cm}^{-1}$ and $1162 \mathrm{~cm}^{-1}$. Typical bands of COL-1 are amide I and amide II peaks which were observed at $1630 \mathrm{~cm}^{-1}$ and $1545 \mathrm{~cm}^{-1}$. Spectra of PCL/COL-1 membranes and PDA coated PCL/COL-1 membranes included stretching around $1645 \mathrm{~cm}^{-1}$ for amide I, $1540 \mathrm{~cm}^{-1}$ for amide II, $1724 \mathrm{~cm}^{-1}$ for carbonyl groups and $1167 \mathrm{~cm}^{-1}$ for C-O groups. These data thus reflected the successful generation of PCL/COL-1 hybrid nanofibers. Figure 2F-P showed that only the nanofibers in PCL membranes exhibited hydrophobic characteristics, whereas the other nanofibers exhibited hydrophilic properties.

\section{Identification of TSPCs}

TSPCs formed colonies after in vitro culture for 10 days as indicated by crystal violet staining (Figure $3 \mathrm{~A}$ and B). They showed fibroblast-like cell morphology in vitro (Figure 3C and D). The FACS results showed that over $99 \%$ of fibroblastic marker CD90 and $88 \%$ of MSCs marker CD44 were positive for TSPCs (Figure 3E and F). And they were negative for the hematopoietic stem cell marker CD34 and the endothelial cell marker CD31 (Figure $3 \mathrm{G}$ and $\mathrm{H}$ ). 


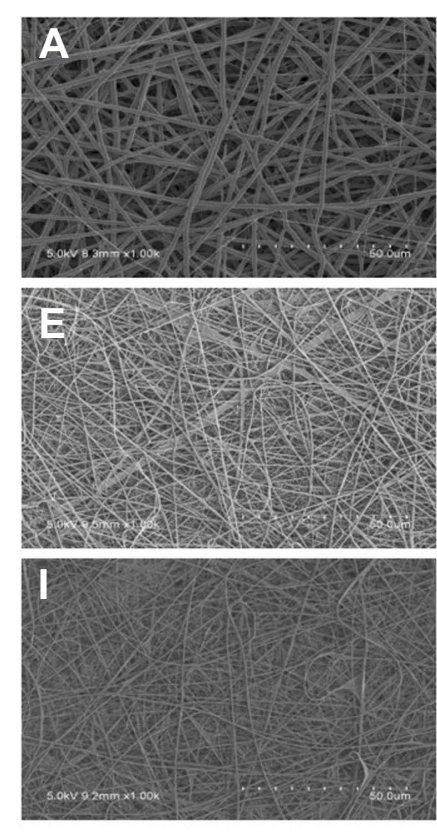

Porosity \%

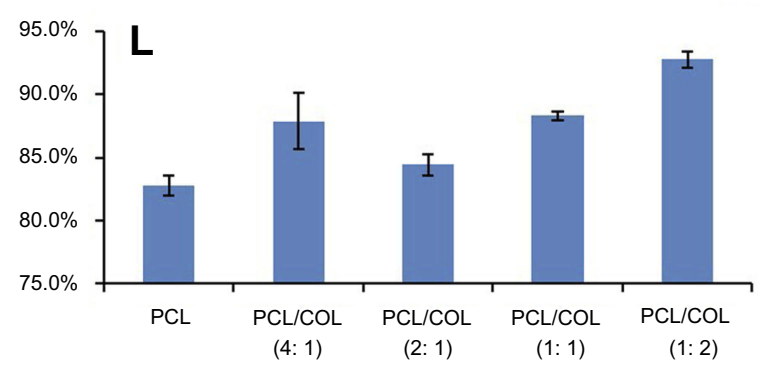

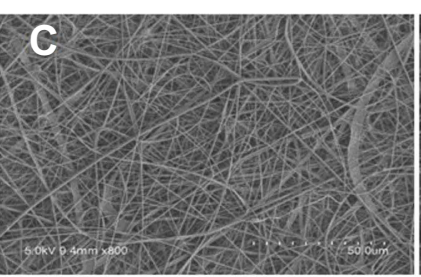
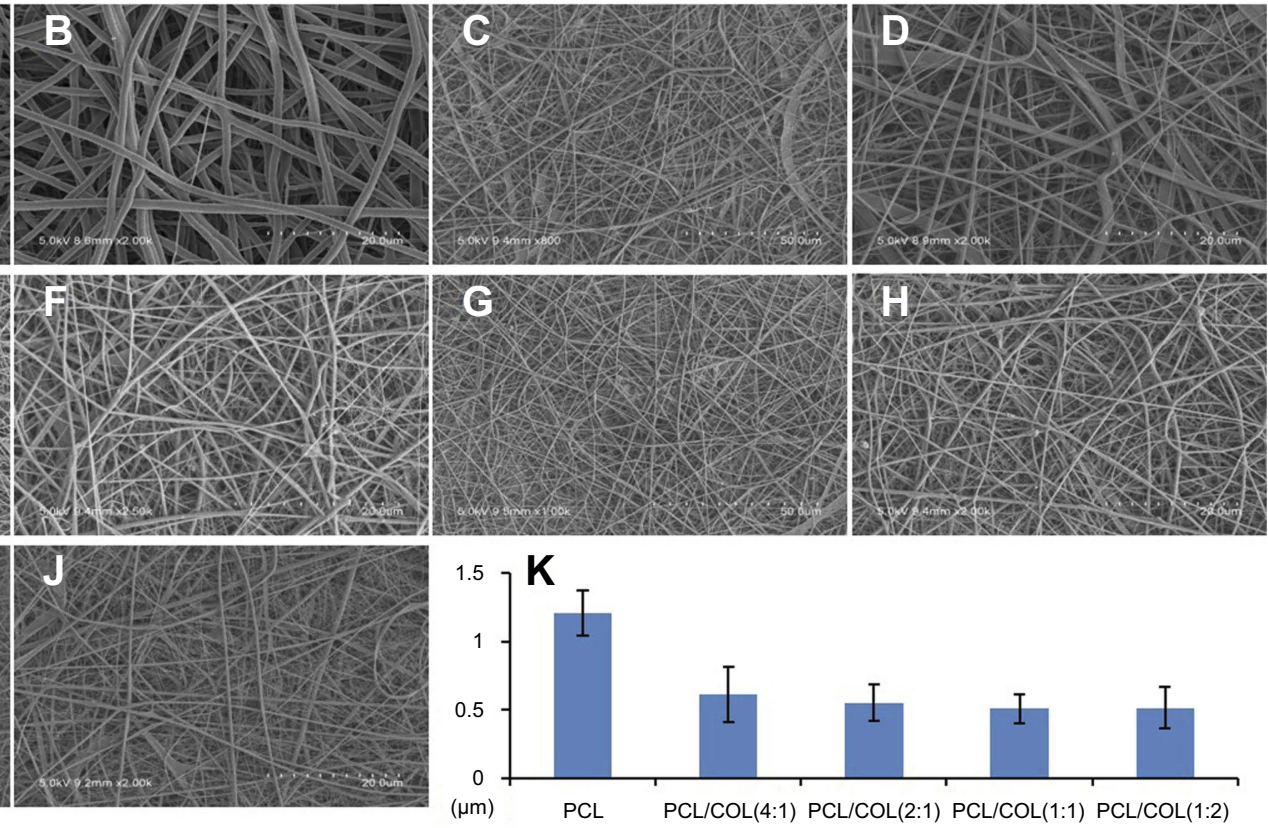

Dissolution rate \%

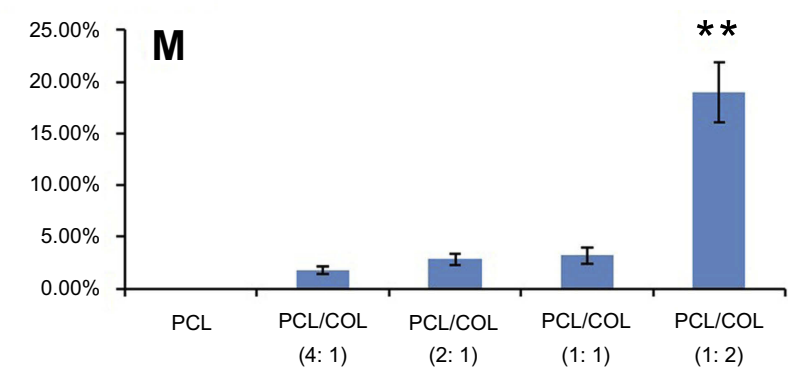

Figure I Morphological, physical and chemical properties analysis of nanofiber membranes. Scanning electron microscopic observation of electrospun nanofiber membranes: (A, C, E, G, I) representing PCL, PCL/COL (4:I), PCL/COL (2:I), PCL/COL (I:I), PCL/COL (I:2) respectively, scale bars: $50 \mu \mathrm{m} ;(\mathbf{B}, \mathbf{D}, \mathbf{F}, \mathbf{H}, \mathbf{J})$ representing PCL, PCL/COL (4:I), PCL/COL (2:I), PCL/COL (I:I), PCL/COL (I:2) respectively, scale bars: $20 \mu \mathrm{m}$. (K) Analysis of the diameters of nanofibers. (L) Porosity measurements using mercury intrusion porosimetry technique. (M) Dissolution rate detection of nanofiber membranes, $* * 0<0.01$.

Abbreviations: PCL, polycaprolactone; $\mathrm{COL}$, collagen.

TSPCs could be induced to differentiate into osteoblasts, adipocytes, and chondrocytes in vitro (Figure 3I-O). The TSPCs exhibited classical MSCs characteristics including clonogenicity, typical surface antigen expression, and multi-lineage differentiation potential.

\section{Cytocompatibility of nanofiber membranes}

To assess the capacity of different scaffolds to support cell migration and function, the proliferation of TSPCs cultured on each material was estimated by the CCK- 8 assay (Figure 4). In the early stages of culture, the positive control group (cells cultured in 96 well plates) had the highest growth rate of all cells $(P<0.05)$. After 3 days, cell proliferation on PCL/COL-1 hybrid group became significantly higher than PCL group
$(P<0.05)$. As culture proceeded, the PCL/COL-1(2:1) group overtook the positive control group $(P<0.05)$ and had the highest optical density value after 5 and 7 days culture.

Figure $5 \mathrm{~A}-\mathrm{R}$ showed representative confocal microscopic images of the results of the live/dead assay. We found there were not many red-stained dead cells in each group. Furthermore, the number of green-stained viable cells was the largest in PCL/COL-1(2:1) group compared to other groups in vitro culture at day $1,3,5$ timepoint.

\section{Cell morphology in nanofiber membranes}

SEM morphologies of TSPCs cultured on PCL membranes, PCL/COL-1 membranes and PDA-modified nanofiber surfaces for various times were shown in Figure 6A-R. TSPCs on all the membranes displayed 

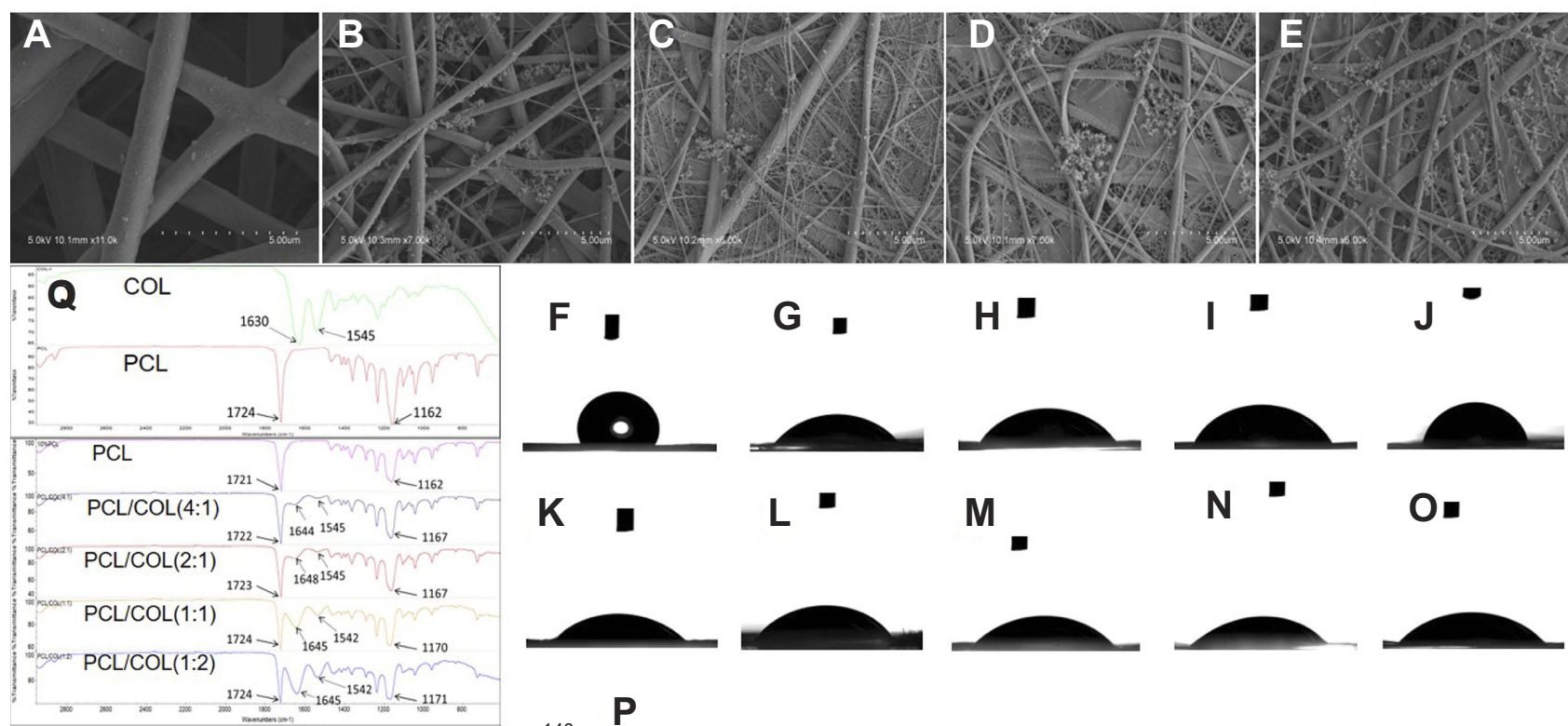

H

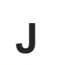

K

L
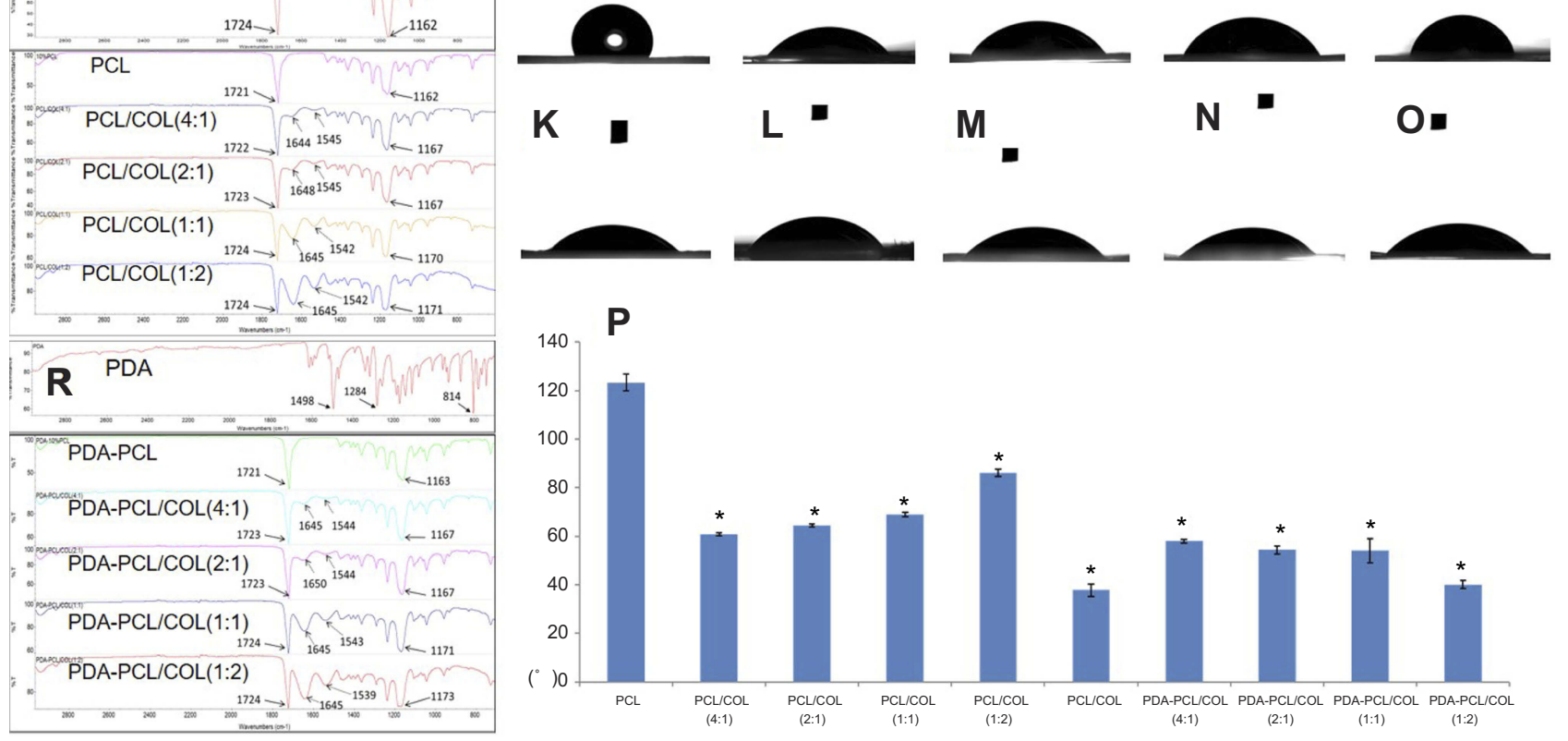

M N 0.

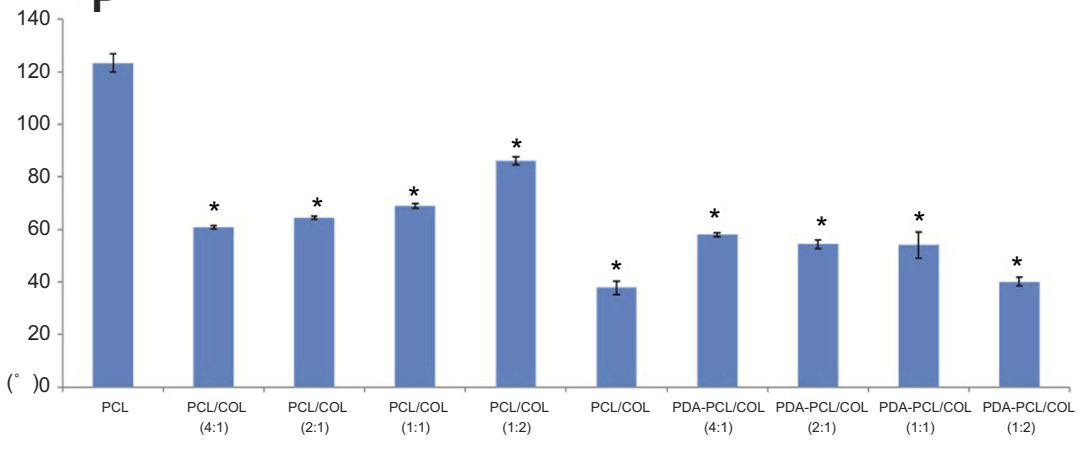

Figure 2 Composition analysis and hydrophilicity detection of nanofiber membranes. Scanning electron microscopic observation of PDA surface coating nanofiber membranes: (A) PDA-coated PCL, (B) PDA-coated PCL/COL (4:I), (C) PDA-coated PCL/COL (2:I), (D) PDA-coated PCL/COL (I:I), (E) PDA-coated PCL/COL (I:2), scale bars: $5 \mu \mathrm{m}$. Water contact angles of nanofiber membranes: (F) PCL, (G) PCL/COL (4:I), (H) PCL/COL (2:I), (I) PCL/COL (I:I), (J) PCL/COL (I:2), (K) PDA-coated PCL, (L) PDA-coated PCL/COL (4:I), (M) PDA-coated PCL/COL (2:I), (N) PDA-coated PCL/COL (I:I), (O) PDA-coated PCL/COL (I:2). (P) Statistical analysis of water contact angle values, ${ }^{*} p<0.05$ compared with $\mathrm{PCL}$ group. $(\mathbf{Q}, \mathbf{R})$ Fourier-transform infrared analysis of nanofiber membranes.

Abbreviations: $\mathrm{PCL}$, polycaprolactone; COL, collagen; PDA, poly(dopamine).

a flat spreading with lots of lamellipodia at day1, and all the samples were covered by extracellular matrix secreted by TSPCs after 3 days culture. There was no obvious difference between the samples.

We compared cell morphology and the organization of actin structures on nanofibers with different surfaces after $24 \mathrm{~h}$ of seeding (Figure 7A-F). Cells grown on unmodified PCL nanofibers displayed a small spherical or narrow shape of TSPCs. On a PCL and COL-1 hybrid electrospun surface, TSPCs were more widely spread than on PCL electrospun fibers and PDA-coated nanofibers. In addition, the area of the projected cells on the PCL/ COL-1(2:1) was significantly greater than the cells on PCL group $(P<0.05)$ (Figure 7G).

\section{Differentiation study of TSPCs in nanofiber membranes}

To assess the effect of nanofiber membranes on TSPCs differentiation, expression of osteogenic genes (Colla1, Ocn, Runx-2) and stem cell marker gene (Oct-4) was quantified after 4, 7 and 14 days of culture (Figure $8 \mathrm{~A}-\mathrm{L}$ ). The expression level of Col1a1 in PCL/COL-1(2:1) membranes was significantly higher than PCL membranes at all time points $(P<0.05)$. No difference of Ocn expression was detected at 4 and 7 days between PCL/COL-1(2:1) and the PCL group, but Ocn expression in the PCL/COL-1(2:1) group showed a significant upregulation at 14 days of culture $(P<0.05)$. Runx-2 expression was significantly increased in PCL/COL-1(2:1) and PCL/COL-1(4:1) groups 

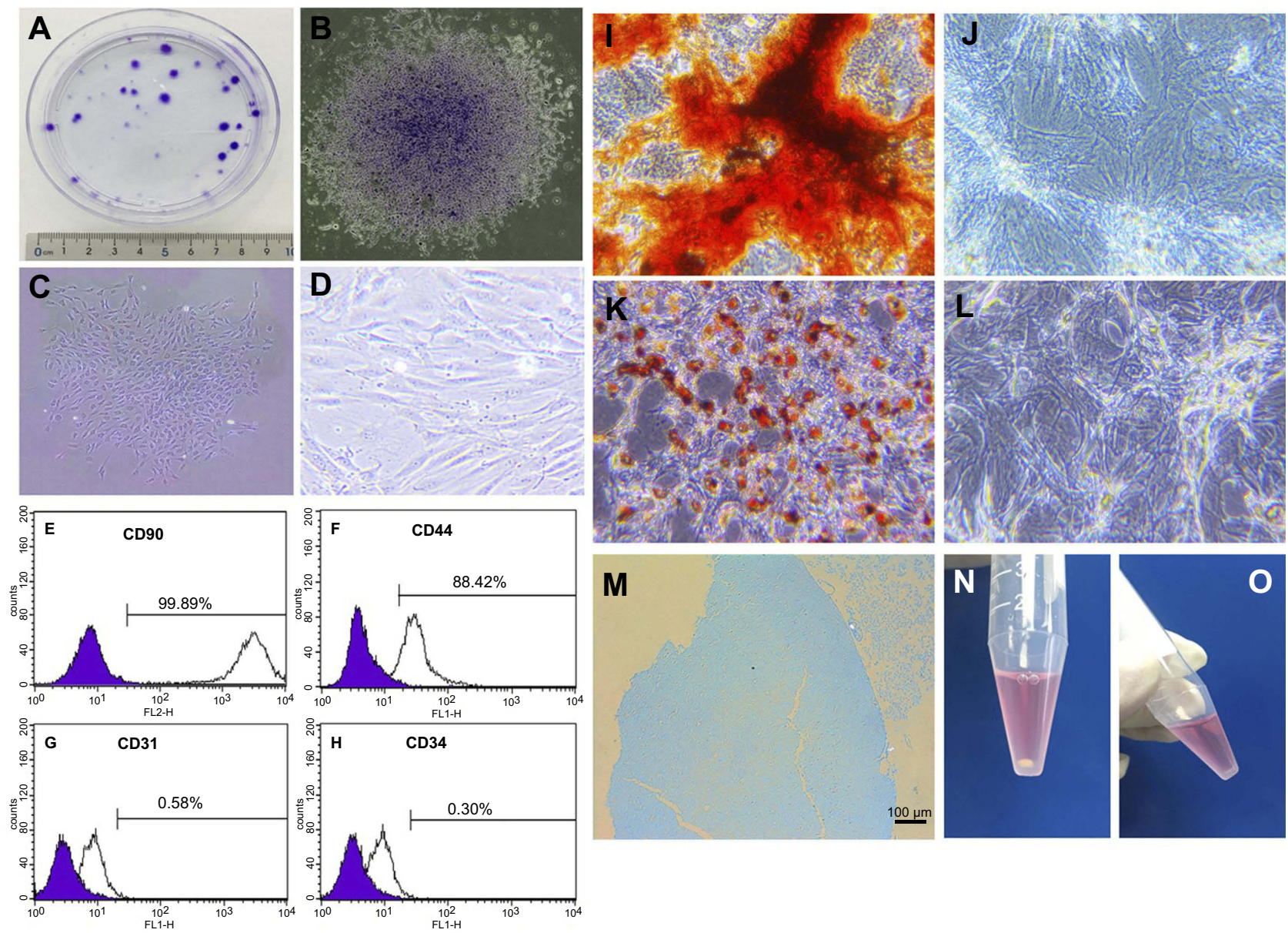

Figure 3 Identification of TSPCs in vitro. (A) General view of colony formation assay. (B) Microscopic observation of single colony colonies with crystal violet staining, magnification: x40. (C) Microscopic observation of P0 cells, magnification: x40. (D) Microscopic observation of P3 cells, magnification: $x$ I00. (E-H) Flow cytometry assay of stem cell surface specific markers. Alizarin red staining in the osteogenic induction group $(\mathbf{I})$ and the control group (J), magnification: $x \mid 00$. Oil red $O$ staining in the adipogenic induction group (K) and the control group (L), magnification: xl00. Alcian blue staining of the cell pellet in the chondrogenic induction group (M), scale bars: $100 \mu \mathrm{m}$. General view of the cell pellet in the chondrogenic induction group $(\mathbf{N})$ and the control group $(\mathbf{O})$.

Abbreviations: TSPCs, tendon stem/progenitor cells.

as compared to PCL fibers at days 4 and $7(p<0.05)$. In contrast, there were no significant differences in Oct-4 expression in PCL/COL-1(2:1) membranes as compared to the PCL membranes at 4 days after culture. After 7 and 14 days of culture, Oct-4 levels were significantly lower in PCL/COL-1(2:1) and PCL/COL-1(4:1) membranes compared to the PCL membranes $(p<0.05)$. Notably, PDAcoated nanofiber membranes could not promote higher osteogenic gene expression than the PCL membranes.

Enhanced osteogenesis of TSPCs in the PCL and COL-1 hybrid electrospun nanofiber membranes was confirmed by immunostaining for osteogenic specific markers. As shown in Figure 9A-R and Figure 10A-R, the expression of Col1 a2 and Ocn, biochemical markers for bone formation, was found more widely distributed in the extracellular region in PCL/COL-1
(2:1) membranes than other groups at 21 days after culture. Moreover, the immunofluorescence pictures (Figure 11A-R) also showed that Runx-2, a key transcription factor of osteoblast differentiation, was expressed higher in PCL/COL-1(2:1) membranes compared with other membranes after 21 days culture.

\section{Discussion}

Osteointegration of the tendon graft in the bone tunnel is thought to be more physiological and beneficial, rather than indirect healing through Sharpey-like fibers. ${ }^{6}$ This study was designed to fabricate a suitable nanofiber membrane and act as a biocompatible scaffold to promote osteogenic regeneration of the tendon to bone interface. In the present study, PCL and COL-1 hybrid 


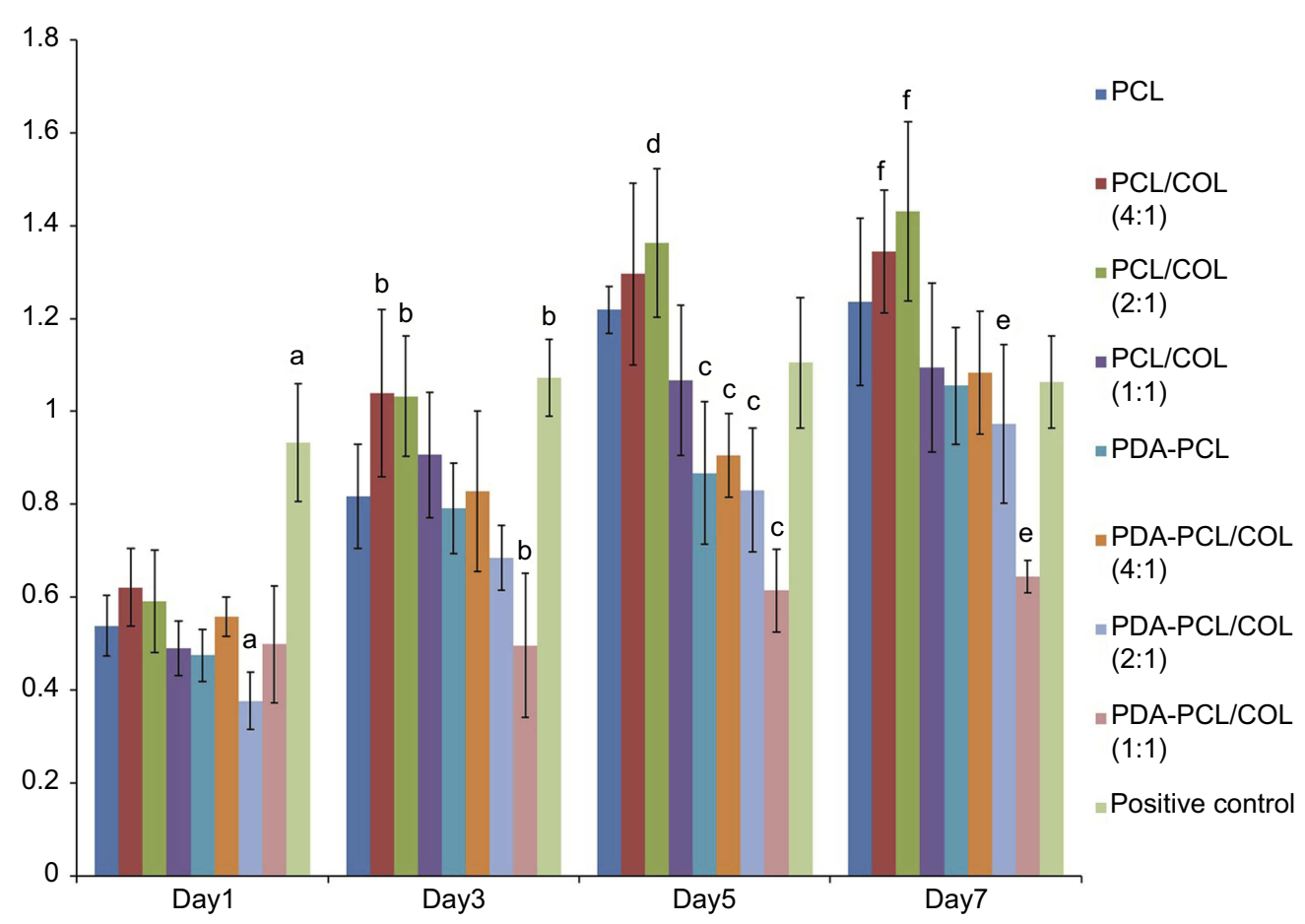

Figure 4 Cell proliferation of TSPCs using cell counting kit-8 assay. ${ }^{a, b, c, e} \mathrm{P}<0.05$ compared with $\mathrm{PCL}$; ${ }^{\mathrm{d}, \mathrm{f}} \mathrm{p}<0.05$ compared with positive control. Abbreviations: TSPCs, tendon stem/progenitor cells; PCL, polycaprolactone; COL, collagen; PDA, poly(dopamine).

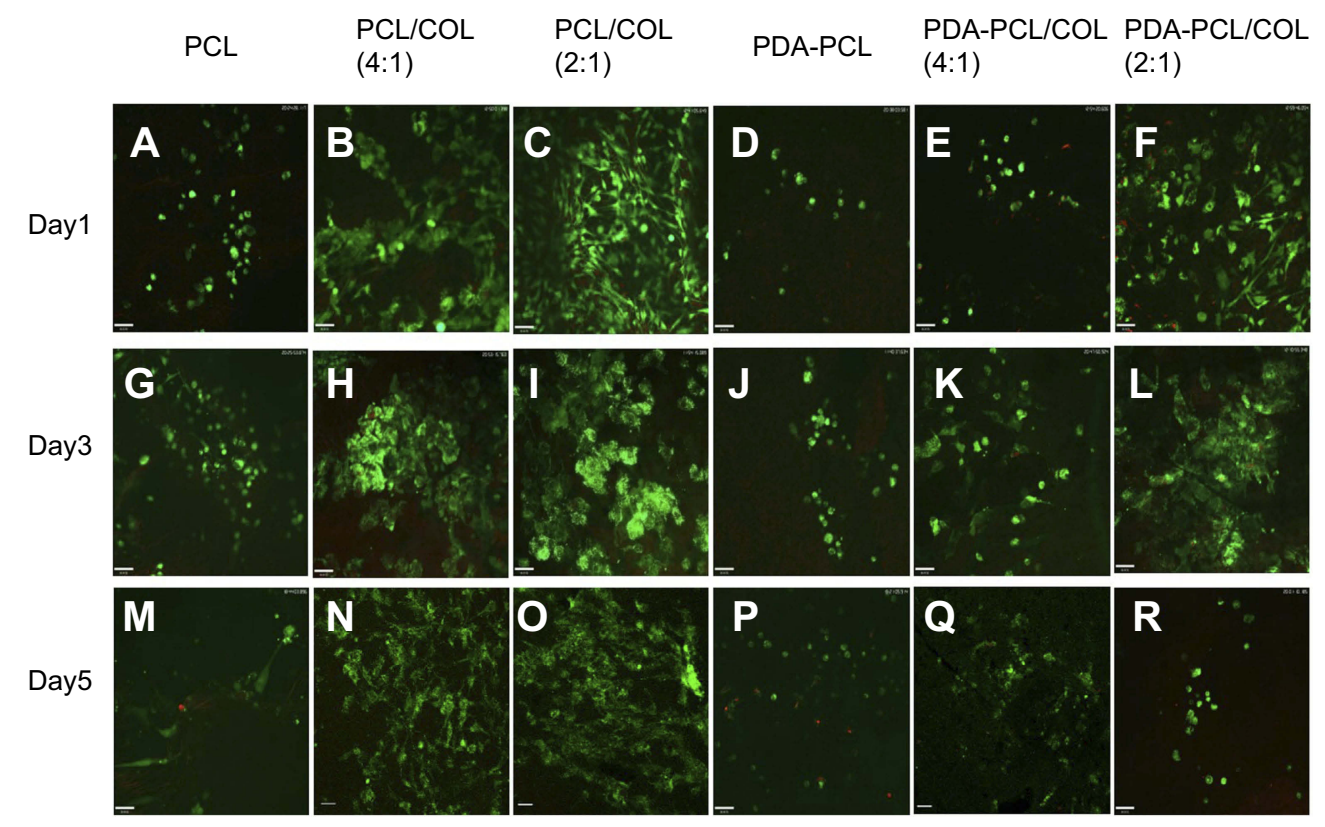

Figure 5 Cell viability of TSPCs in nanofiber membranes using the Live/Dead Viability assay. (A, G, M) TSPCs cultured on PCL for dayl, day3, day5. (B, H, N) TSPCs cultured on PCL/COL (4:I) for dayl, day3, day5. (C, I, O) TSPCs cultured on PCL/COL (2:I) for dayl, day3, day5. (D, J, P) TSPCs cultured on PDA-coated PCL for dayl, day3, day5. (E, K, Q) TSPCs cultured on PDA-coated PCL/COL (4:I) for dayl, day3, day5. (F, L, R) TSPCs cultured on PDA-coated PCL/COL (2:I) for dayI, day3, day5, scale bars: $58 \mu \mathrm{m}$.

Abbreviations: TSPCs, tendon stem/progenitor cells; PCL, polycaprolactone; COL, collagen; PDA, poly(dopamine).

electrospun nanofiber membranes (volume ratio: $2: 1 \mathrm{v} / \mathrm{v}$ ) were superior in terms of their ability to support the adhesion, proliferation, and osteogenic differentiation of
TSPCs, representing an ideal bioactive scaffold for biological tendon-bone tissue engineering applications (Figure 12). 


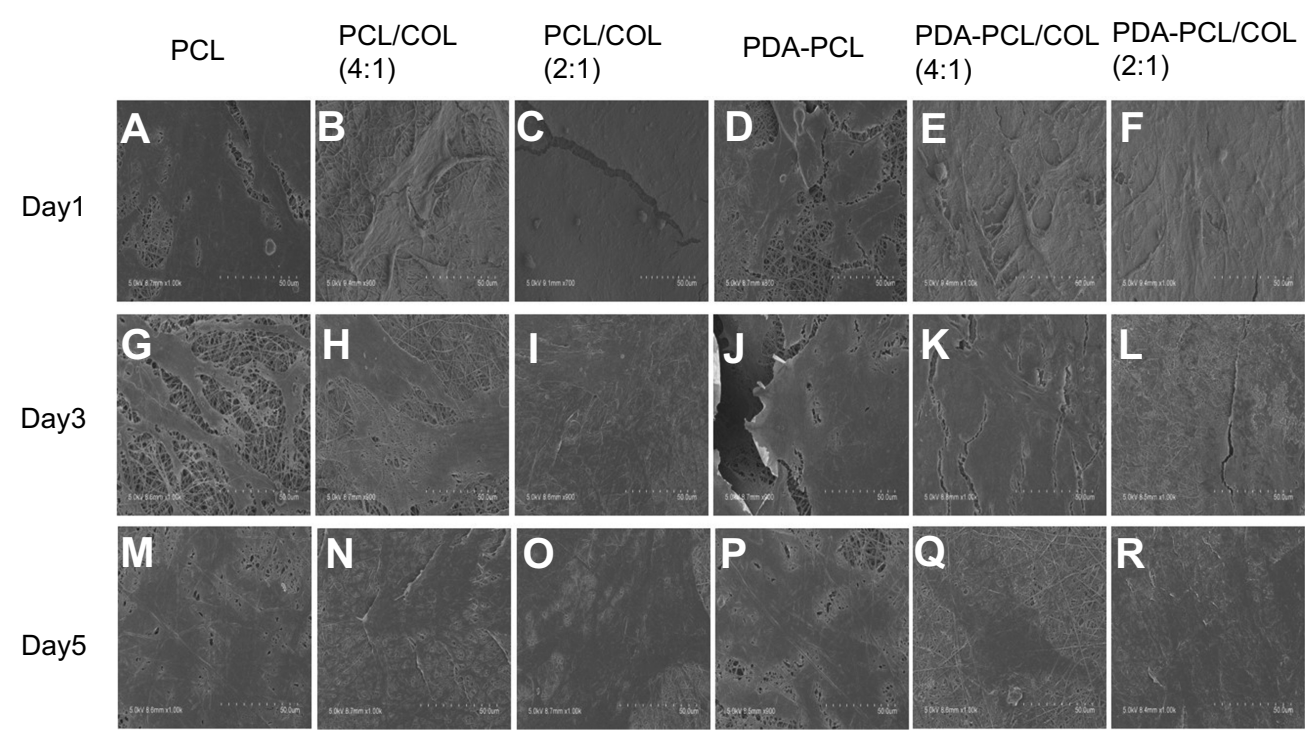

Figure 6 Cell morphology of TSPCs in nanofiber membranes using scanning electron microscopic observation. (A, G, M) TSPCs cultured on PCL for dayl, day3, day5. (B, $\mathbf{H}, \mathbf{N})$ TSPCs cultured on PCL/COL (4:I) for dayl, day3, day5. (C, I, O) TSPCs cultured on PCL/COL (2:I) for dayI, day3, day5. (D, J, P) TSPCs cultured on PDA-coated PCL for dayl, day3, day5. (E, K, Q) TSPCs cultured on PDA-coated PCL/COL (4:I) for dayl, day3, day5. (F, L, R) TSPCs cultured on PDA-coated PCL/COL (2:I) for dayI, day3, day5, scale bars: $50 \mu \mathrm{m}$.

Abbreviations: TSPCs, tendon stem/progenitor cells; PCL, polycaprolactone; COL, collagen; PDA, poly(dopamine).

The ECM microenvironment of the cellular niche is crucial as it can control the behavior and fate of stem cells. ${ }^{36}$ Nanofiber membranes produced by electrospinning are widely used as biomaterials because they can imitate the nanoscale structure and morphology of ECM with controllable fiber diameter and alignment. ${ }^{37}$ Collagen type I fibers are the main component of the ECM of both tendon and bone tissues, exhibiting a porous fibrous network and the orientation of the fibers in bone is random with nanometer-scale diameters. Since the ideal scaffolds probably resemble the structural morphology of the natural collagen found in the target tissues, ${ }^{38}$ the electrospun nanofibers used in this study are similar to the structures of native ECM with random alignment. And these nanofiber membranes exhibited a porous structure with smooth surfaces and few nodes. Furthermore, the average diameters of PCL/COL-1 membranes $(\backsim 500 \mathrm{~nm})$ were considered to simulate the diameters of natural type I collagen fibrils ranging from $50 \mathrm{~nm}$ to $500 \mathrm{~nm}$ (Figure 1K). ${ }^{39}$ As shown in Figure 1L, the nonwoven mat of electrospun nanofibers exhibited high porosity (more than $80 \%$ ) and could thus enhance nutrient delivery and the removal of metabolic wastes. ${ }^{40}$ The dissolvability test showed that PCL/COL-1 (1:2) membranes had gravimetrically $19.04 \%$ weight loss after the first hour of PBS treatment and far exceeding the other groups, which indicated that this ratio of blend solution was unfavorable for tissue engineering applications and need to be excluded.
The successful surface coating of nanofiber membranes using PDA was confirmed by a color change in brown and an increase in the roughness of the membranes. As shown in Figure 2A-E, diameters of the nanofibers did not change after the PDA coating and these results were consistent with other studies which showed unchanged fiber morphology with PDA coating. ${ }^{26}$ What's more, either the combination with PCL and COL-1 polymers or surface modification with PDA could effectively improve the hydrophilicity of the membranes (Figure 2F-P). The hydrophilic feature makes the membranes more suitable for cell adherence and spreading than hydrophobic surfaces. ${ }^{41}$

Although the optical density of TSPCs on all types of membranes increased during the 7 days culture, the proliferation ability of PCL/COL-1 (2:1) group was superior to the others (Figure 4). Additionally, the number of green stained living cells in PCL/COL-1 membranes was also significantly higher than in PCL and PDA coating membranes (Figure 5). The excellent cytocompatibility of PCL/ COL-1 membranes is mainly due to the successful nanofiber functionalization resulted from synergistic effects of PCL and collagen, which is supported by the results of the previous studies. ${ }^{42}$ Our cytoskeleton staining results showed that TSPCs on PCL/COL-1 hybrid membranes had a larger cell projected area than on PCL and PDAmodified membranes, suggesting the better cell adhesion in PCL/COL-1 hybrid scaffolds (Figure 7). Lee et al also 

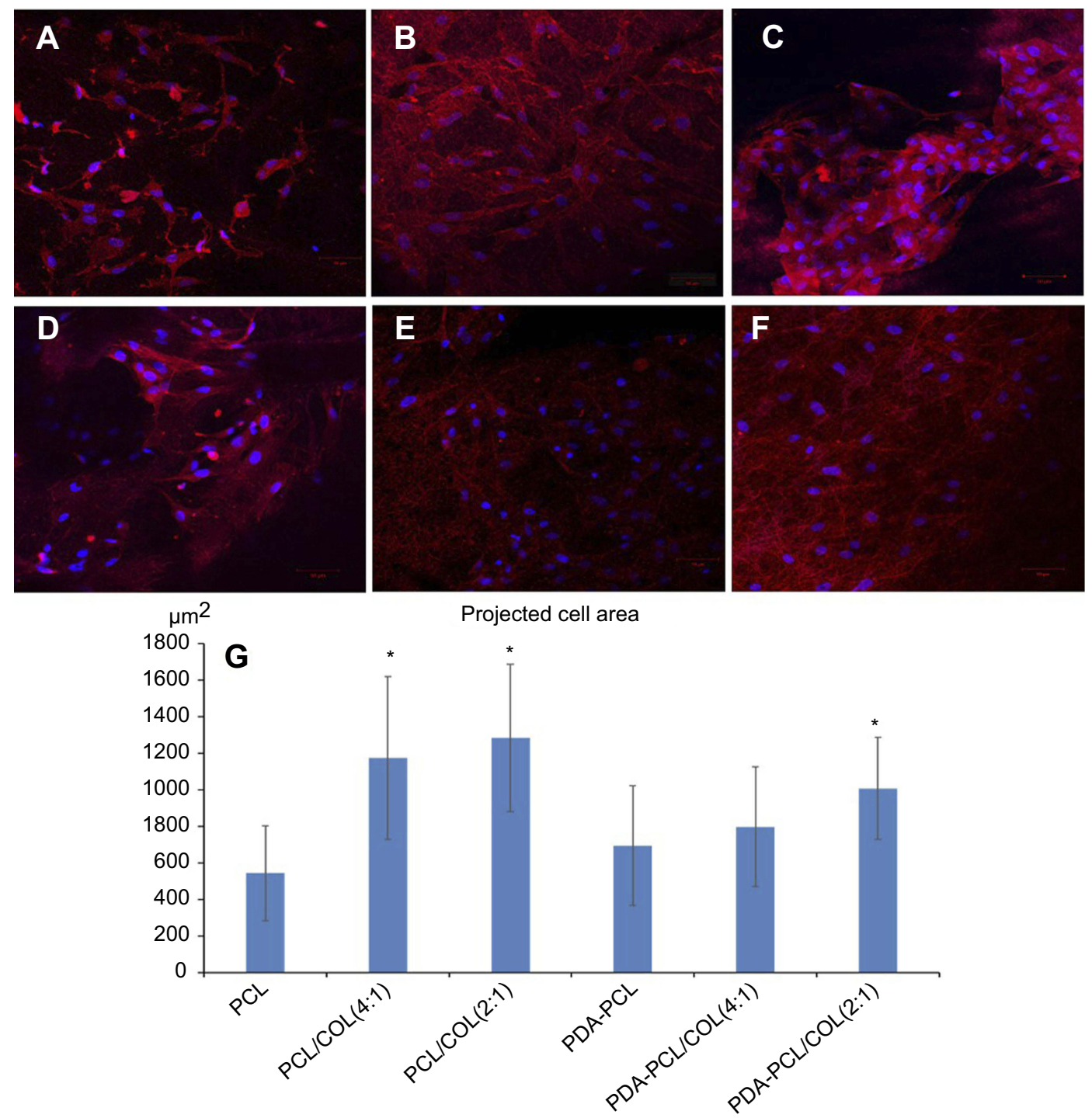

Figure 7 The cytoskeleton morphology of TSPCs using fluorescence microscopy. Fluorescence staining of TSPCs on electrospun nanofibers after $24 \mathrm{~h}$ of culture: (A) PCL, (B) PCL/COL (4:I), (C) PCL/COL (2:I), (D) PDA-coated PCL, (E) PDA-coated PCL/COL (4:I), (F) PDA-coated PCL/COL (2:I), scale bars: 50 $\mu$ m. (G) Projected area per cell, ${ }^{*} p<0.05$ compared with PCL.

Abbreviations: TSPCs, tendon stem/progenitor cells; PCL, polycaprolactone; COL, collagen; PDA, poly(dopamine).

reported that because of the influence by the PDA coating, round-shaped MSCs with the formation of mature filopodia were not observed on PDA-coated random fibers. ${ }^{26}$

Overall, this study showed that PDA coating had no advantages in cell adhesion and proliferation compared with PCL and PCL/COL-1 hybrid membranes. Interestingly, PDA surface modification was found to promote endothelial cells attachment and proliferation, while inhibiting the adhesion and proliferation of smooth muscle cells. ${ }^{43,44}$ They concluded that the effect of PDA coating on cytocompatibility of nanofibers seemed to be cell typedependent. Ding et al suggested that the quinone group on PDA coatings promoted endothelial cells attachment and proliferation, whereas the phenolic hydroxyl groups on PDA coatings would account for the inhibitory effects on smooth muscle cells. ${ }^{45}$ Although many studies have reported the effects of PDA coating on cell adhesion and proliferation of various materials, the polymerization mechanism and the reasons for diverse growth behaviors in different types of cells during PDA modified process are still not very clear. Moreover, very few studies have investigated the effects of PDA modification on TSPCs. It was speculated that the cellular behaviors might be affected by the functional groups like primary amino, catechol, and quinone existing on the PDA surfaces. ${ }^{43}$ Contrary to the enhancement of cell adhesion and 

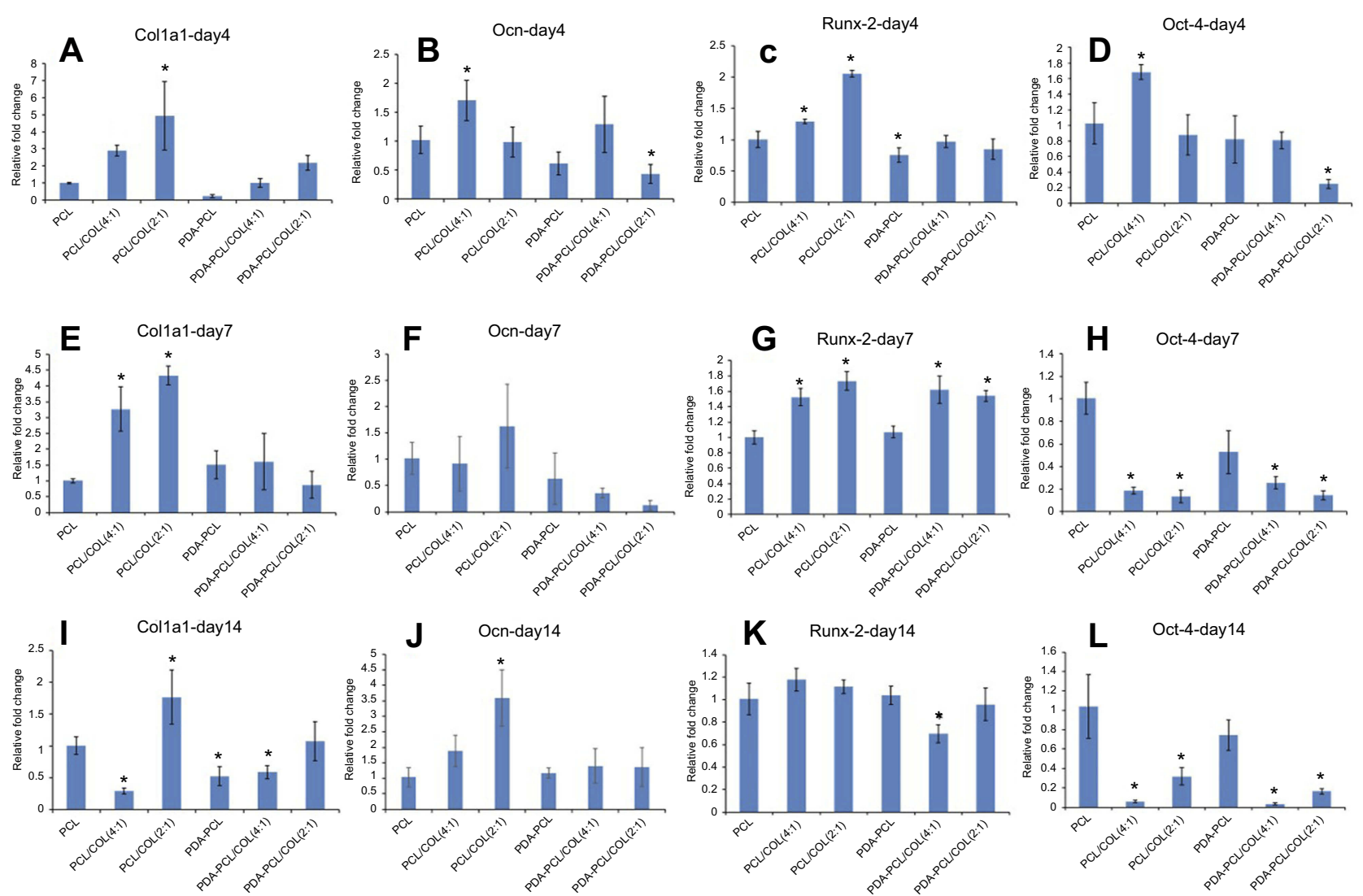

Figure 8 Quantitative real-time reverse transcription polymerase chain reaction (qRT-PCR) for osteogenic differentiation of TSPCs. mRNA expression of Collal in nanofiber membranes for 4 days (A), 7 days (E) and I4 days (I). mRNA expression of Ocn in nanofiber membranes for 4 days (B), 7 days (F) and I4 days (J). mRNA expression of Runx-2 in nanofiber membranes for 4 days $(\mathbf{C}), 7$ days $(\mathbf{G})$ and 14 days (K). mRNA expression of Oct-4 in nanofiber membranes for 4 days $(\mathbf{D}), 7$ days $(\mathbf{H})$ and 14 days $(\mathbf{L})$. $* p<0.05$ compared with PCL.

Abbreviations: TSPCs, tendon stem/progenitor cells; PCL, polycaprolactone; COL, collagen; PDA, poly(dopamine); Colla I, collagen I alpha I; Ocn, osteocalcin.

proliferation in PDA-coated surfaces, the inhibitory effect of PDA coating on adhesion and proliferation of TSPCs might also be associated with the surface catechol content. Further research about how the catechol of PDA affects TSPCs growth is urgently needed.

In addition, the rate of cell proliferation of $\mathrm{PCL} / \mathrm{COL}-1$ (1:1) group was the lowest in different ratios of PCL/COL1 hybrid membranes with or without PDA coating. Therefore, this result suggested that PCL/COL-1 (1:1) group may not be optimal for continued research in tissue engineering applications.

There are many factors that can guide the differentiation of stem cells on nanofiber membranes, including the morphological characteristics of nanofibers and the properties of polymers. ${ }^{46}$ It has been reported that small-diameter $(<1 \mu \mathrm{m})$ electrospun fibers, random orientation morphology, and hybridization of synthetic and natural polymers are strongly associated with osteogenesis. ${ }^{47-49}$ Roman et al showed that MSCs exhibited the greatest osteogenic differentiation when cultured on the tissue culture plates coated with type I collagen compared with the uncoated plates. ${ }^{50}$ They suggested that although the mechanism of how COL-1 affected the osteogenic differentiation of stem cells was unknown, the most significant role in promoting osteogenesis appeared to activate the transcription factor Runx2/CBFA-1 in integrin signaling pathways. Our differentiation results were similar to these previous studies. In this study, PCL/COL-1 hybrid membranes demonstrated superior ability to induce TSPCs osteogenic differentiation than PCL membranes and PDA-coated membranes due to the suitable fiber diameter, random nanotopography, and advantages of hybridizing PCL and COL-1. Importantly, we found that the performance of PCL/COL-1 (2:1) group was the best in the results of cell proliferation, adhesion, and osteogenic differentiation of TSPCs, which indicated that for subsequent tissue engineering studies the optimal volume ratio of PCL to COL- 1 could be 2:1. No benefit was found in this study to the PDA coating on osteogenic 


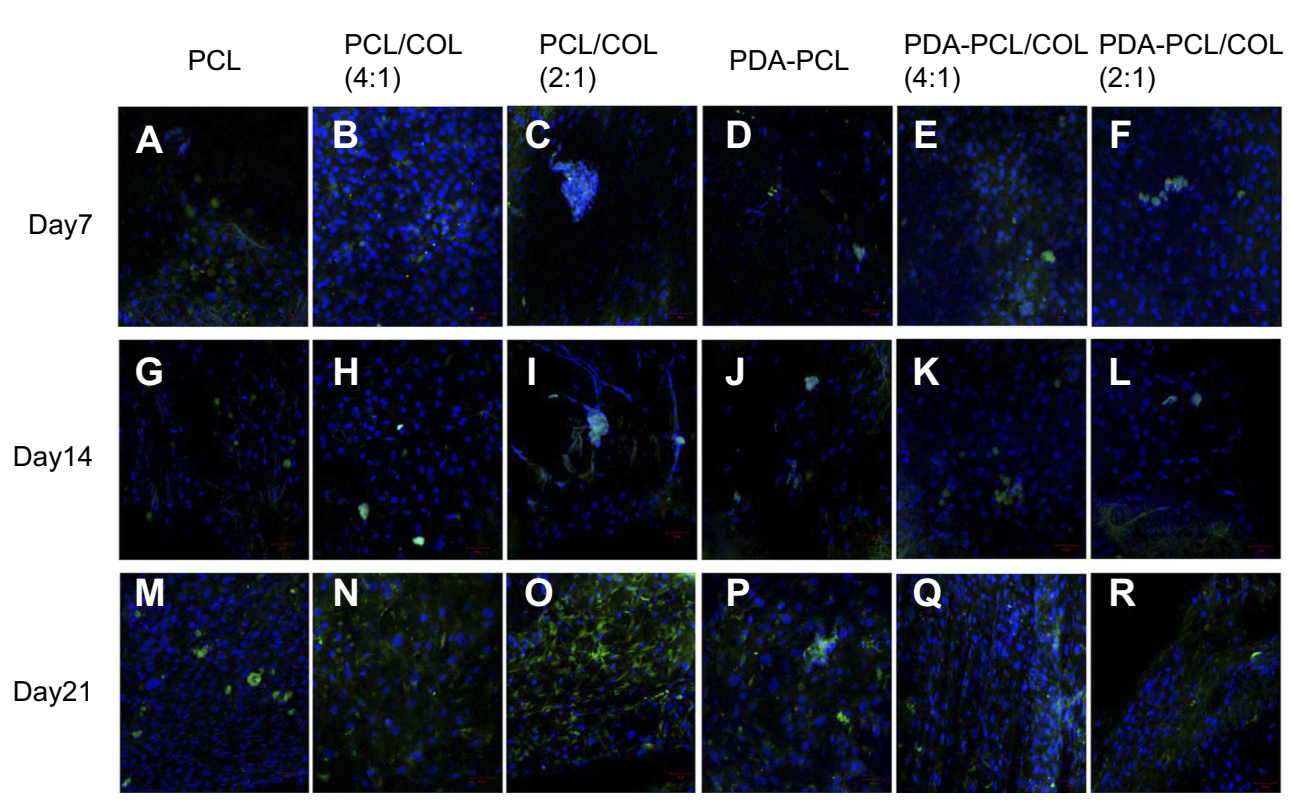

Figure 9 Colla2 expression using immunofluorescent staining assay. (A, G, M) TSPCs cultured on PCL for day7, dayl4, day2 I. (B, H, N) TSPCs cultured on PCL/ COL (4:I) for day7, dayl4, day2I. (C, I, O) TSPCs cultured on PCL/COL (2:I) for day7, dayl4, day2I. (D,J,P) TSPCs cultured on PDA-coated PCL for day7, dayl4, day2I. (E, K, Q) TSPCs cultured on PDA-coated PCL/COL (4:I) for day7, dayl4, day2I. (F, L, R) TSPCs cultured on PDA-coated PCL/COL (2:I) for day7, dayl4, day2 I, scale bars: $50 \mu \mathrm{m}$.

Abbreviations: TSPCs, tendon stem/progenitor cells; PCL, polycaprolactone; COL, collagen; PDA, poly(dopamine); Colla2, collagen I alpha 2.

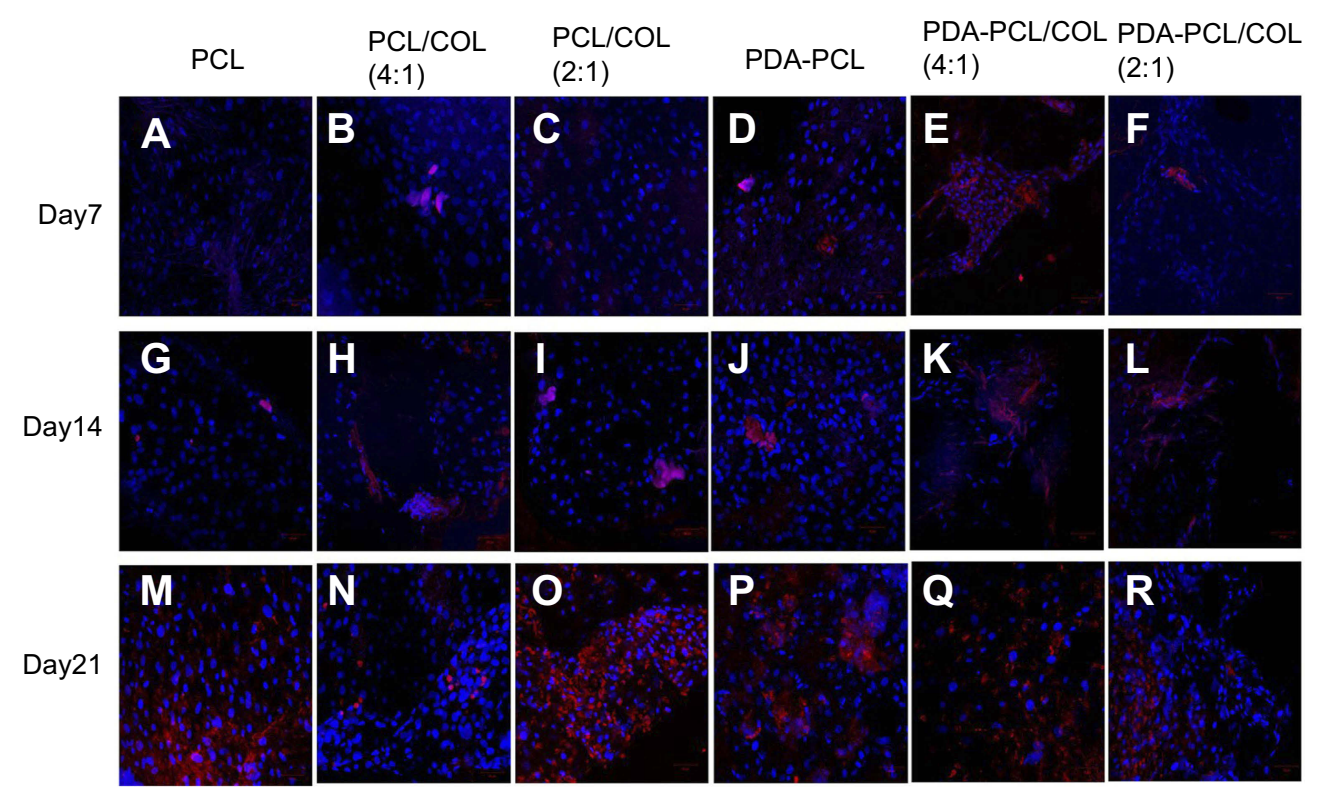

Figure 10 Ocn expression using immunofluorescent staining assay. (A, G, M) TSPCs cultured on PCL for day7, dayl4, day2 I. (B, H, N) TSPCs cultured on PCL/COL (4:I) for day7, dayl4, day2I. (C, I, O) TSPCs cultured on PCL/COL (2:I) for day7, dayl4, day2I. (D, J, P) TSPCs cultured on PDA-coated PCL for day7, dayl4, day2I. (E, K, Q) TSPCs cultured on PDA-coated PCL/COL (4:I) for day7, dayl4, day2I. (F, L, R) TSPCs cultured on PDA-coated PCL/COL (2:I) for day7, dayl4, day2I, scale bars: 50 $\mu$ m. Abbreviations: TSPCs, tendon stem/progenitor cells; PCL, polycaprolactone; COL, collagen; PDA, poly(dopamine); Ocn, osteocalcin.

differentiation of TSPCs. A similar conclusion was found in a previous study showing that PDA deposition did not enhance the differentiated phenotype of osteoblasts. ${ }^{25}$ The effect of PDA coating on cell differentiation is also controversial. The PDA-coated nanofibrous substrates demonstrated remarkable effects in stimulating osteogenic differentiation of human MSCs and adipose-derived stem cells compared to the original materials, ${ }^{51,52}$ whereas Lee reported that there were no observed changes in osteogenic markers between PDA treated and pristine Ti alloys. ${ }^{53}$ 


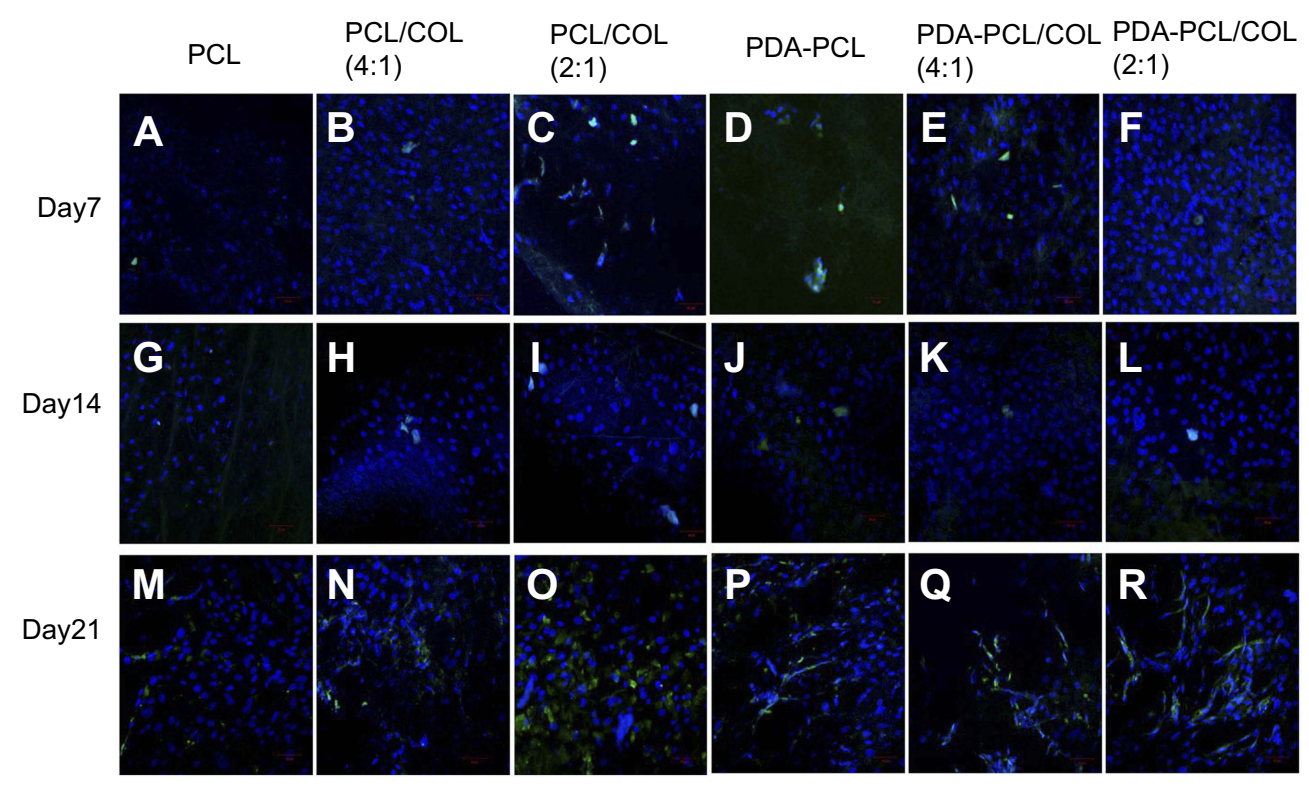

Figure II Runx-2 expression using immunofluorescent staining assay. (A, G, M) TSPCs cultured on PCL for day7, day 14, day2I. (B, H, N) TSPCs cultured on PCL/COL (4:I) for day7, day 14, day2I. (C, I, O) TSPCs cultured on PCL/COL (2:I) for day7, day 14, day21. (D, J, P) TSPCs cultured on PDA-coated PCL for day7, day 14, day21. (E, K, Q) TSPCs cultured on PDA-coated PCL/COL (4:I) for day7, day 14, day2I. (F, L, R) TSPCs cultured on PDA-coated PCL/COL (2:I) for day7, day 14, day2I, scale bars: $50 \mu \mathrm{m}$. Abbreviations: TSPCs, tendon stem/progenitor cells; PCL, polycaprolactone; COL, collagen; PDA, poly(dopamine).

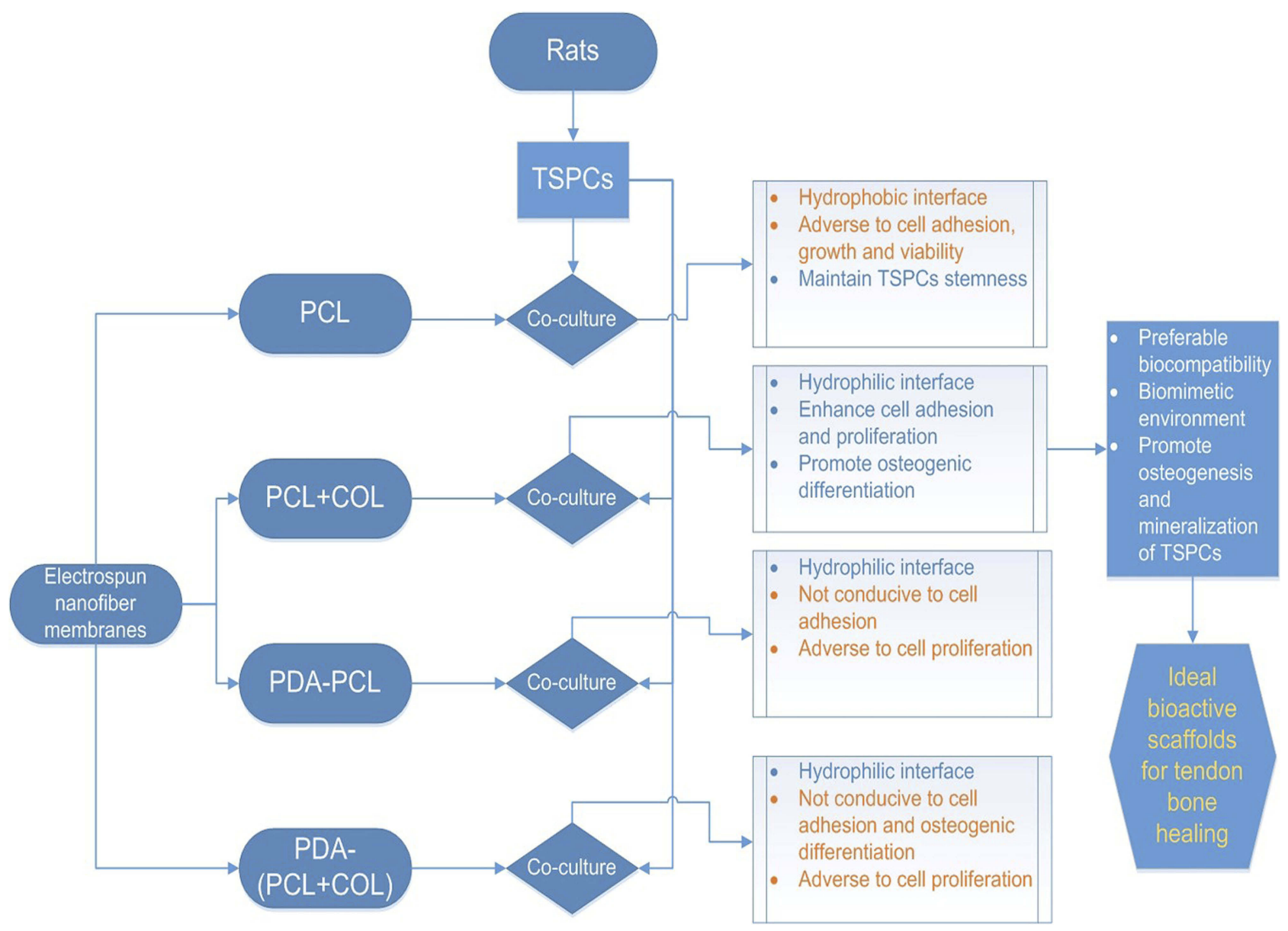

Figure 12 Schematic diagram of the regulation of electrospun nanofiber membranes on TSPCs.

Abbreviations: TSPCs, tendon stem/progenitor cells; PCL, polycaprolactone; COL, collagen; PDA, poly(dopamine). 
Furthermore, a deleterious effect on the osteogenic activity of MC3T3-E1 cells after PDA coating of the nanostructured surface was observed in a recent study. ${ }^{54}$ Although we chose the most widely used PDA coating method, which was first introduced by Lee in $2007,{ }^{22}$ many features of PDA formation and structure are still unknown. The contradictory results about the diverse response of cell differentiation to PDA-coated nanofibers could derive from the different properties of the fibers on which PDA is deposited, the time elapsed from PDA deposition, the reaction temperature, initial PDA concentration, the solution $\mathrm{pH}$, and the oxygen concentration in the PDA solution. ${ }^{54}$ Therefore, for a better understanding of PDAcoated surface-modified scaffolds impact on cell behavior more in-depth studies are required.

Although the use of synthetic scaffold of PCL and COL-1 as a base material presents the advantage of reasonable and controlled biodegradation rate which matches regeneration rate of the target tissues, ${ }^{40}$ degradation experiment for a longer period of time needs to be further studied in vitro. Besides, the promotion of these scaffolds for tendon-bone healing needs to be further investigated in an in vivo tendon-bone healing animal model in the future.

\section{Conclusion}

In this study, pure PCL nanofiber membranes and PCL/COL-1 hybrid membranes were successfully fabricated by electrospinning. And then these nanofiber membranes were coated with PDA as the simple surface modification. Furthermore, the membranes were seeded with TSPCs to assess the impact of electrospun fibers on cellular behavior and differentiation characteristics. Our results exhibit that the presence of PDA coating on these fibers does not show the benefits of promoting cell adhesion, projected cell area, cell proliferation, and osteogenic differentiation. The PCL/COL-1 hybrid membranes are demonstrated to be more biocompatible, biomimetic and are able to support cell adhesion, proliferation, and osteogenic differentiation in vitro. Therefore, the bioactive PCL/COL-1 hybrid membranes may be an appropriate scaffold for tendonbone tissue engineering applications.

\section{Acknowledgments}

This work was financially supported by National Natural Science Foundation of China (81771985 and 81572188), Key Research Program of Science and Technology of Jiangsu Province (BE2015613) and Nanjing Science and Technology Development Foundation (201805001).

\section{Disclosure}

The authors report no conflicts of interest in this work.

\section{References}

1. Lomas AJ, Ryan CN, Sorushanova A, et al. The past, present and future in scaffold-based tendon treatments. Adv Drug Deliv Rev. 2015;84:257-277. doi:10.1016/j.addr.2014.11.022

2. von Porat A, Roos EM, Roos H. High prevalence of osteoarthritis 14 years after an anterior cruciate ligament tear in male soccer players: a study of radiographic and patient relevant outcomes. Ann Rheum Dis. 2004;63(3):269-273. doi:10.1136/ard.2003.008136

3. Han F, Zhang P, Sun Y, Lin C, Zhao P, Chen J. Hydroxyapatite-doped polycaprolactone nanofiber membrane improves tendon-bone interface healing for anterior cruciate ligament reconstruction. Int $J$ Nanomedicine. 2015;10:7333-7343. doi:10.2147/IJN.S92099

4. Ekdahl M, Wang JH, Ronga M, Fu FH. Graft healing in anterior cruciate ligament reconstruction. Knee Surg Sports Traumatol Arthrosc. 2008;16(10):935-947. doi:10.1007/s00167-008-0584-0

5. Park MJ, Lee MC, Seong SC. A comparative study of the healing of tendon autograft and tendon-bone autograft using patellar tendon in rabbits. Int Orthop. 2001;25(1):35-39. doi:10.1007/ s002640000199

6. Park SH, Choi YJ, Moon SW, et al. Three-dimensional bio-printed scaffold sleeves with mesenchymal stem cells for enhancement of tendon-to-bone healing in anterior cruciate ligament reconstruction using soft-tissue tendon graft. Arthroscopy. 2018;34(1):166-179. doi:10.1016/j.arthro.2017.04.016

7. Weimin P, Dan L, Yiyong W, Yunyu H, Li Z. Tendon-to-bone healing using an injectable calcium phosphate cement combined with bone xenograft/BMP composite. Biomaterials. 2013;34(38):9926-9936. doi:10.1016/j.biomaterials.2013.09.018

8. Oh JH, Chung SW, Kim SH, Chung JY, Kim JY. 2013 Neer Award: effect of the adipose-derived stem cell for the improvement of fatty degeneration and rotator cuff healing in rabbit model. $J$ Shoulder Elbow Surg. 2014;23(4):445-455. doi:10.1016/j. jse.2013.07.054

9. Mifune Y, Matsumoto T, Takayama K, et al. Tendon graft revitalization using adult anterior cruciate ligament (ACL)-derived CD34+ cell sheets for ACL reconstruction. Biomaterials. 2013;34(22):54765487. doi:10.1016/j.biomaterials.2013.04.013

10. Chang $\mathrm{CH}$, Chen $\mathrm{CH}$, Su CY, Liu HT, Yu CM. Rotator cuff repair with periosteum for enhancing tendon-bone healing: a biomechanical and histological study in rabbits. Knee Surg Sports Traumatol Arthrosc. 2009;17(12):1447-1453. doi:10.1007/s00167-009-0809-x

11. Moffat KL, Kwei AS, Spalazzi JP, Doty SB, Levine WN, Lu HH. Novel nanofiber-based scaffold for rotator cuff repair and augmentation. Tissue Eng Part A. 2009;15(1):115-126. doi:10.1089/ten. tea.2008.0014

12. Atesok K, Fu FH, Wolf MR, et al. Augmentation of tendon-to-bone healing. J Bone Joint Surg Am. 2014;96(6):513-521. doi:10.2106/ JBJS.M.00009

13. Cai J, Wang J, Ye K, et al. Dual-layer aligned-random nanofibrous scaffolds for improving gradient microstructure of tendon-to-bone healing in a rabbit extra-articular model. Int $J$ Nanomedicine. 2018;13:3481-3492. doi:10.2147/IJN.S165633

14. Li X, Xie J, Lipner J, Yuan X, Thomopoulos S, Xia Y. Nanofiber scaffolds with gradations in mineral content for mimicking the tendon-to-bone insertion site. Nano Lett. 2009;9(7):2763-2768. doi: $10.1021 / \mathrm{n} 1901582 \mathrm{f}$

15. Chou YC, Yeh WL, Chao CL, et al. Enhancement of tendon-bone healing via the combination of biodegradable collagen-loaded nanofibrous membranes and a three-dimensional printed bone-anchoring bolt. Int $J$ Nanomedicine. 2016;11:4173-4186. doi:10.2147/IJN. S108939 
16. Cao D, Wu YP, Fu ZF, et al. Cell adhesive and growth behavior on electrospun nanofibrous scaffolds by designed multifunctional composites. Colloids Surf B Biointerfaces. 2011;84(1):26-34. doi:10.1016/j.colsurfb.2010.12.005

17. Malikmammadov E, Tanir TE, Kiziltay A, Hasirci V, Hasirci N. PCL and PCL-based materials in biomedical applications. J Biomater Sci Polym Ed. 2018;29(7-9):863-893. doi:10.1080/09205063.2017.1394711

18. Barnes CP, Sell SA, Boland ED, Simpson DG, Bowlin GL. Nanofiber technology: designing the next generation of tissue engineering scaffolds. Adv Drug Deliv Rev. 2007;59(14):1413-1433. doi:10.1016/j. addr.2007.04.022

19. Gui-Bo Y, You-Zhu Z, Shu-Dong W, De-Bing S, Zhi-Hui D, WeiGuo F. Study of the electrospun PLA/silk fibroin-gelatin composite nanofibrous scaffold for tissue engineering. $J$ Biomed Mater Res A. 2010;93(1):158-163. doi:10.1002/jbm.a.32496

20. Gautam S, Dinda AK, Mishra NC. Fabrication and characterization of PCL/gelatin composite nanofibrous scaffold for tissue engineering applications by electrospinning method. Mater Sci Eng C Mater Biol Appl. 2013;33(3):1228-1235. doi:10.1016/j.msec.2012.12.015

21. Gumusderelioglu M, Dalkiranoglu S, Aydin RS, Cakmak S. A novel dermal substitute based on biofunctionalized electrospun PCL nanofibrous matrix. J Biomed Mater Res A. 2011;98(3):461-472. doi:10.1002/jbm.a.33143

22. Lee H, Dellatore SM, Miller WM, Messersmith PB. Mussel-inspired surface chemistry for multifunctional coatings. Science. 2007;318 (5849):426-430. doi:10.1126/science.1147241

23. Yang K, Lee JS, Kim J, et al. Polydopamine-mediated surface modification of scaffold materials for human neural stem cell engineering. Biomaterials. 2012;33(29):6952-6964. doi:10.1016/j. biomaterials.2012.06.067

24. Chuah YJ, Koh YT, Lim K, Menon NV, Wu Y, Kang Y. Simple surface engineering of polydimethylsiloxane with polydopamine for stabilized mesenchymal stem cell adhesion and multipotency. Sci Rep. 2015;5:18162. doi:10.1038/srep 18162

25. Tsai WB, Chen WT, Chien HW, Kuo WH, Wang MJ. Poly(dopamine) coating to biodegradable polymers for bone tissue engineering. J Biomater Appl. 2014;28(6):837-848. doi:10.1177/ 0885328213483842

26. Lee JH, Lee YJ, Cho HJ, Shin H. Guidance of in vitro migration of human mesenchymal stem cells and in vivo guided bone regeneration using aligned electrospun fibers. Tissue Eng Part A. 2014;20(1516):2031-2042. doi:10.1089/ten.tea.2013.0282

27. Bi Y, Ehirchiou D, Kilts TM, et al. Identification of tendon stem/ progenitor cells and the role of the extracellular matrix in their niche. Nat Med. 2007;13(10):1219-1227. doi:10.1038/nm1630

28. Zhang J, Wang JH. Characterization of differential properties of rabbit tendon stem cells and tenocytes. BMC Musculoskelet Disord. 2010;11:10. doi:10.1186/1471-2474-11-10

29. Rui YF, Lui PP, Li G, Fu SC, Lee YW, Chan KM. Isolation and characterization of multipotent rat tendon-derived stem cells. Tissue Eng Part A. 2010;16(5):1549-1558. doi:10.1089/ten.TEA.2009.0529

30. Lovati AB, Corradetti B, Lange Consiglio A, et al. Characterization and differentiation of equine tendon-derived progenitor cells. $J$ Biol Regul Homeost Agents. 2011;25(2 Suppl):S75-S84.

31. Lui PP, Wong OT. Tendon stem cells: experimental and clinical perspectives in tendon and tendon-bone junction repair. Muscles Ligaments Tendons J. 2012;2(3):163-168.

32. Tan Q, Lui PP, Rui YF, Wong YM. Comparison of potentials of stem cells isolated from tendon and bone marrow for musculoskeletal tissue engineering. Tissue Eng Part A. 2012;18(7-8):840-851. doi:10.1089/ten.TEA.2011.0362

33. Manoukian OS, Matta R, Letendre J, Collins P, Mazzocca AD, Kumbar SG. Electrospun nanofiber scaffolds and their hydrogel composites for the engineering and regeneration of soft tissues. Methods Mol Biol. 2017;1570:261-278. doi:10.1007/978-1-4939-6840-4_18
34. Zuo Y, Ye G. Pore structure characterization of sodium hydroxide activated slag using mercury intrusion porosimetry, nitrogen adsorption, and image analysis. Materials (Basel). 2018;11(6). doi:10.3390/ ma11081451

35. Lin YC, Li YJ, Rui YF, et al. The effects of high glucose on tendonderived stem cells: implications of the pathogenesis of diabetic tendon disorders. Oncotarget. 2017;8(11):17518-17528. doi:10.18632/ oncotarget. 15418

36. Lutolf MP, Gilbert PM, Blau HM. Designing materials to direct stemcell fate. Nature. 2009;462(7272):433-441. doi:10.1038/nature08602

37. Khorshidi S, Solouk A, Mirzadeh H, et al. A review of key challenges of electrospun scaffolds for tissue-engineering applications. $J$ Tissue Eng Regen Med. 2016;10(9):715-738. doi:10.1002/term.1978

38. Schumann D, Ekaputra AK, Lam CX, Hutmacher DW. Biomaterials/scaffolds. Design of bioactive, multiphasic PCL/collagen type I and type II-PCL-TCP/collagen composite scaffolds for functional tissue engineering of osteochondral repair tissue by using electrospinning and FDM techniques. Methods Mol Med. 2007;140:101-124.

39. Gelse K, Poschl E, Aigner T. Collagens-structure, function, and biosynthesis. Adv Drug Deliv Rev. 2003;55(12):1531-1546.

40. Li WJ, Laurencin CT, Caterson EJ, Tuan RS, Ko FK. Electrospun nanofibrous structure: a novel scaffold for tissue engineering. $J$ Biomed Mater Res. 2002;60(4):613-621.

41. Wen J, Lu T, Wang X, et al. In vitro and in vivo evaluation of silicatecoated polyetheretherketone fabricated by electron beam evaporation. ACS Appl Mater Interfaces. 2016;8(21):13197-13206. doi:10.1021/ acsami.5b10229

42. Zhang Q, Lv S, Lu J, Jiang S, Lin L. Characterization of polycaprolactone/collagen fibrous scaffolds by electrospinning and their bioactivity. Int J Biol Macromol. 2015;76:94-101. doi:10.1016/j. ijbiomac.2015.01.063

43. Luo R, Tang L, Zhong S, et al. In vitro investigation of enhanced hemocompatibility and endothelial cell proliferation associated with quinone-rich polydopamine coating. ACS Appl Mater Interfaces. 2013;5(5):1704-1714. doi:10.1021/am3027635

44. Yang Z, Tu Q, Zhu Y, et al. Mussel-inspired coating of polydopamine directs endothelial and smooth muscle cell fate for re-endothelialization of vascular devices. Adv Healthc Mater. 2012;1(5):548-559. doi:10.1002/adhm.201200073

45. Ding YH, Floren M, Tan W. Mussel-inspired polydopamine for biosurface functionalization. Biosurf Biotribol. 2016;2(4):121-136. doi:10.1016/j.bsbt.2016.11.001

46. Xue J, Xie J, Liu W. Electrospun nanofibers: new concepts, materials, and applications. Acc Chem Res. 2017;50(8):1976-1987. doi:10.1021/acs.accounts.7b00218

47. Ekaputra AK, Zhou Y, Cool SM, Hutmacher DW. Composite electrospun scaffolds for engineering tubular bone grafts. Tissue Eng Part A. 2009;15(12):3779-3788. doi:10.1089/ten.TEA.2009.0186

48. Cardwell RD, Dahlgren LA, Goldstein AS. Electrospun fibre diameter, not alignment, affects mesenchymal stem cell differentiation into the tendon/ligament lineage. J Tissue Eng Regen Med. 2014;8 (12):937-945. doi:10.1002/term.1589

49. Yin Z, Chen X, Chen JL, et al. The regulation of tendon stem cell differentiation by the alignment of nanofibers. Biomaterials. 2010;31 (8):2163-2175. doi:10.1016/j.biomaterials.2009.11.083

50. Salasznyk RM, Williams WA, Boskey A, Batorsky A, Plopper GE. Adhesion to vitronectin and collagen I promotes osteogenic differentiation of human mesenchymal stem cells. J Biomed Biotechnol. 2004;2004(1):24-34. doi:10.1155/S1110724304306017

51. Rim NG, Kim SJ, Shin YM, et al. Mussel-inspired surface modification of poly(L-lactide) electrospun fibers for modulation of osteogenic differentiation of human mesenchymal stem cells. Colloids Surf B Biointerfaces. 2012;91:189-197. doi:10.1016/j. colsurfb.2011.10.057 
52. Lin CC, Fu SJ. Osteogenesis of human adipose-derived stem cells on poly (dopamine)-coated electrospun poly(lactic acid) fiber mats. Mater Sci Eng C Mater Biol Appl. 2016;58:254-263. doi:10.1016/j.msec.2015.08.009

53. Lee JH, Lim YW, Kwon SY, Kim YS. In vitro effects of musselinspired polydopamine coating on Ti6A14V alloy. Tissue Eng Regen Med. 2013;10(5):273-278. doi:10.1007/s13770-012-1089-y
54. Albu AM, Draghicescu W, Munteanu T, et al. Nitrodopamine vs dopamine as an intermediate layer for bone regeneration applications. Mat Sci Eng C-Mater. 2019;98:461-471. doi:10.1016/j. msec.2019.01.014

\section{Publish your work in this journal}

The International Journal of Nanomedicine is an international, peerreviewed journal focusing on the application of nanotechnology in diagnostics, therapeutics, and drug delivery systems throughout the biomedical field. This journal is indexed on PubMed Central, MedLine, CAS, SciSearch ${ }^{\mathbb{R}}$, Current Contents ${ }^{\mathbb{R}} /$ Clinical Medicine,
Journal Citation Reports/Science Edition, EMBase, Scopus and the Elsevier Bibliographic databases. The manuscript management system is completely online and includes a very quick and fair peer-review system, which is all easy to use. Visit http://www.dovepress.com/ testimonials.php to read real quotes from published authors. 